\title{
Lecithin:Cholesterol Acyltransferase Activation by Sulfhydryl-Reactive Small Molecules: Role of Cysteine-31 ${ }^{\mathrm{s}}$
}

\author{
Lita A. Freeman, Stephen J. Demosky, Jr., Monika Konaklieva, Rostislav Kuskovsky, \\ Angel Aponte, Alice F. Ossoli, Scott M. Gordon, Ross F. Koby, Kelly A. Manthei, Min Shen, \\ Boris L. Vaisman, Robert D. Shamburek, Ajit Jadhav, Laura Calabresi, Marjan Gucek, \\ John J.G. Tesmer, Rodney L. Levine, and Alan T. Remaley
}

Lipid Metabolism Section, Cardiovascular and Pulmonary Branch (L.A.F., S.J.D., S.M.G., B.L.V., R.D.S., A.T.R.), Systems Biology Center (A.A., M.G.), and Laboratory of Biochemistry (R.L.L.), National Institutes of Health National Heart, Lung, and Blood Institute, Bethesda, Maryland; Department of Chemistry, American University, Washington, DC (M.K., R.K.); University of Milano, Milano, Italy (A.F.O., L.C.); Department of Chemistry, Vanderbilt University, Nashville, Tennessee (R.F.K.); Departments of Pharmacology and Biological Chemistry, Life Sciences Institute, University of Michigan, Ann Arbor, Michigan (K.A.M., J.J.G.T.); and National Institutes of Health National Center for Advancing Translational Sciences, Bethesda, Maryland (M.S., A.J.)

Received January 28, 2017; accepted April 19, 2017

\section{ABSTRACT}

Lecithin:cholesterol acyltransferase (LCAT) catalyzes plasma cholesteryl ester formation and is defective in familial lecithin: cholesterol acyltransferase deficiency (FLD), an autosomal recessive disorder characterized by low high-density lipoprotein, anemia, and renal disease. This study aimed to investigate the mechanism by which compound A [3-(5-(ethylthio)-1,3,4-thiadiazol2-ylthio)pyrazine-2-carbonitrile], a small heterocyclic amine, activates LCAT. The effect of compound A on LCAT was tested in human plasma and with recombinant LCAT. Mass spectrometry and nuclear magnetic resonance were used to determine compound $A$ adduct formation with LCAT. Molecular modeling was performed to gain insight into the effects of compound A on LCAT structure and activity. Compound A increased LCAT activity in a subset (three of nine) of LCAT mutations to levels comparable to FLD heterozygotes. The site-directed mutation LCAT-Cys31Gly prevented activation by compound A. Substitution of Cys31 with charged residues (Glu, Arg, and Lys) decreased LCAT activity, whereas bulky hydrophobic groups (Trp, Leu, Phe, and Met) increased activity up to 3-fold ( $P<$ 0.005). Mass spectrometry of a tryptic digestion of LCAT incubated with compound A revealed a $+103.017 \mathrm{~m} / \mathrm{z}$ adduct on Cys31, consistent with the addition of a single hydrophobic cyanopyrazine ring. Molecular modeling identified potential interactions of compound A near Cys31 and structural changes correlating with enhanced activity. Functional groups important for LCAT activation by compound A were identified by testing compound A derivatives. Finally, sulfhydryl-reactive $\beta$-lactams were developed as a new class of LCAT activators. In conclusion, compound A activates LCAT, including some FLD mutations, by forming a hydrophobic adduct with Cys31, thus providing a mechanistic rationale for the design of future LCAT activators.

\section{Introduction}

High-density lipoprotein cholesterol (HDL-C) levels in plasma are inversely related to cardiovascular disease (CVD) risk, but most therapeutic approaches for increasing HDL-C in clinical trials have not shown benefit (Rader, 2016). It may be the function rather than the cholesterol content of high-density lipoprotein (HDL) that accounts for its atheroprotection

This research was supported [in part] by the Intramural Research Program of the National Institutes of Health [National Heart, Lung, and Blood Institute and National Center for Advancing Translational Sciences]. This research was also supported by the National Institutes of Health National Heart, Lung, and Blood Institute [Grants R01-HL071818 and R01-HL122416 (to J.J.G.T.) and Ruth L. Kirschstein National Research Service Award F32HL131288 (to K.A.M.)].

https://doi.org/10.1124/jpet.117.240457.

S This article has supplemental material available at jpet.aspetjournals.org.
(Karathanasis et al., 2017). HDLs are a complex mixture of particles with many different protein and lipid components. Protective functions ascribed to HDL include reverse cholesterol transport from aortic foam cells to the liver, as well as antiinflammatory, antioxidative, and antiprotease effects (Rye and Barter, 2014; Rosenson et al., 2016; Gordon and Remaley, 2017). Many of these functions of HDLs are performed by larger, spherical particles, so maturation of HDLs from small discoidal particles to large spherical particles may be important for CVD protection (Asztalos et al., 2000, 2011; Karathanasis et al., 2017).

Lecithin:cholesterol acyltransferase (LCAT), a plasma protein secreted by the liver, is an enzyme critical for HDL particle maturation (Ahsan et al., 2014). LCAT catalyzes cholesteryl ester (CE) production from free cholesterol (FC)

ABBREVIATIONS: CE, cholesteryl ester; CER, cholesterol esterification rate; CVD, cardiovascular disease; DMSO, dimethylsulfoxide; DTNB, dithiobis-nitrobenzoic acid; DTT, dithiothreitol; FC, free cholesterol; FLD, familial lecithin:cholesterol acyltransferase deficiency; GSH, glutathione; $\mathrm{HDL}$, high-density lipoprotein; HDL-C, high-density lipoprotein cholesterol; LC, liquid chromatography; LCAT, lecithin:cholesterol acyltransferase; MS, mass spectrometry; msrA, methionine sulfoxide reductase A; MUP, methylumbelliferyl palmitate; m/z, mass-to-charge ratio; NMR, nuclear magnetic resonance; rhLCAT, recombinant human lecithin:cholesterol acyltransferase; WT, wild type. 
and phosphatidylcholine (lecithin). Because CEs are more hydrophobic than FC, LCAT activity promotes partitioning of cholesterol from the surface of HDL into its core, converting small discoidal HDLs to larger, spherical HDL particles. Moreover, esterification promotes the net egress of cholesterol from cells because unlike FC, CE does not freely equilibrate between cells and lipoproteins. Esterification thereby makes cholesterol efflux to HDL irreversible (Czarnecka and Yokoyama, 1996). Once formed, CE is preferentially delivered to the liver (Schwartz et al., 2004).

CE production by LCAT takes place in two steps. First, LCAT cleaves fatty acids from the $s n-2$ position of phospholipids, using its phospholipase A2 activity. Ser181, part of the catalytic triad of LCAT (Ser181, Asp345, His377), acts as the nucleophile and is acylated during this step (Ahsan et al., 2014). Next, LCAT transfers the cleaved fatty acid from Ser181 to the A-ring hydroxyl group of cholesterol to produce CE. Phosphatidylcholines are the preferred phospholipid substrate, particularly those with unsaturated fatty acids in the sn-2 position (Ahsan et al., 2014).

Recent X-ray crystallographic studies of LCAT confirm the presence of a flexible lid (residues 226-246) that may alter accessibility of the catalytic site to its lipid substrates (Glukhova et al., 2015; Piper et al., 2015; Gunawardane et al., 2016). Certain lid residues (227-DNQGIPVMSS-236) may also contribute to substrate specificity (Glukhova et al., 2015). Residues 36-101 comprise the membrane binding domain of LCAT (Glukhova et al., 2015), with residues 50-74 forming part of the HDL/low-density lipoprotein interfacial binding domain (Adimoolam and Jonas, 1997). LCAT has six cysteines, four in disulfide bonds (Cys50-Cys74 and Cys313Cys356) that are critical for LCAT activity (Glukhova et al., 2015). The two free cysteines (Cys31 and Cys184) are near the active site, with the Cys31 backbone amide forming part of the oxyanion hole (Holleboom et al., 2011; Glukhova et al., 2015).

Many different LCAT mutations have been described in human patients (Ahsan et al., 2014). The most severe mutations result in familial lecithin:cholesterol acyltransferase deficiency (FLD), characterized by low HDL-C, corneal opacities, anemia, and proteinuria that typically progresses by age $40-50$ years to end-stage renal disease, the primary cause of morbidity and mortality. LpX, an abnormal multivesicular-like lipoprotein that accumulates in patients with FLD, contributes to renal disease (Ossoli et al., 2016). Less severe LCAT mutations lead to fish-eye disease, characterized by low HDL-C and corneal opacities but none of the other clinical features of FLD. LCAT is also secondarily decreased in an interleukin6-dependent pathway under some conditions (Feister et al., 2002), likely contributing to decreased HDL during inflammation (Khovidhunkit et al., 2004). Decreased LCAT activity has also been observed in "disappearing HDL syndrome" in several disorders associated with extremely high levels of interleukin-10 (Moraitis et al., 2015).

We recently developed recombinant human lecithin:cholesterol acyltransferase (rhLCAT) as a potential therapy and showed that it safely increased HDL-C levels in both patients with coronary artery disease and individuals with FLD (Shamburek et al., 2016a,b). Because rhLCAT raises HDL and enhances cholesterol efflux, it might protect against CVD, particularly acute coronary syndrome, similar to findings with reconstituted HDL in early-stage clinical trials (Remaley et al., 2008, 2014; Krause and Remaley, 2013). rhLCAT therapy, however, will likely be relatively expensive and require intravenous infusion. An oral small-molecule activator that increases activity in patients with FLD with some residual activity is a possible alternative to rhLCAT therapy and would also be a preferable treatment for CVD. Compound A [3-(5-(ethylthio)1,3,4-thiadiazol-2-ylthio)pyrazine-2-carbonitrile)], the only known small-molecule LCAT activator, was discovered by Amgen in a high-throughput screen (Kayser et al., 2013) and was shown to increase plasma CE and HDL-C levels in mice and hamsters, hepatic Abcg5/g8 and Cyp $7 a 1$ gene expression in mice, and sterol biliary excretion in hamsters (Chen et al., 2012).

Our objective was to investigate the mechanism for LCAT activation by compound $\mathrm{A}$ and to determine whether it can also increase activity of some natural LCAT mutations. We found that compound A can indeed activate a subset of FLD mutations by forming a hydrophobic adduct with Cys31 near the active site. We also identified functional groups in compound A responsible for LCAT activation, and we describe a new class of LCAT activators based on sulfhydryl-reactive $\beta$-lactams. Our findings provide important new mechanistic insights into LCAT activation that can be used to design novel LCAT activators.

\section{Materials and Methods}

Cholesterol Esterification Rate. Human plasma for these studies was obtained under informed consent from protocols approved by the National Institutes of Health National Heart, Lung, and Blood Institute Institutional Review Board and the Milano Area C Ethics Committee. The cholesterol esterification rate (CER) was determined by the rate of esterification of $\left[{ }^{3} \mathrm{H}\right]$ cholesterol, using $125 \mu \mathrm{l}$ of plasma samples before and after incubation of plasma at $37^{\circ} \mathrm{C}$ for 1 hour. Before starting the reaction, plasma from subjects with LCAT deficiency carrying different mutations was incubated with compound $\mathrm{A}$ at a final concentration of $10 \mu \mathrm{g} / \mathrm{ml}$. The reaction was stopped with cold ethanol, cholesterol was extracted, and CE was separated from FC by Thin-layer chromatography (TLC) (Dobiásová, 1983; Dobiásová and Schützová, 1986; Vaisman and Remaley, 2013).

Alternatively, CER was quantitated in some plasma from Italian carriers of LCAT deficiency by measuring FC content before and after incubation of plasma at $37^{\circ} \mathrm{C}$ for 1 hour by an enzymatic colorimetric method (Kempen et al., 2016). A conversion factor between the radioactive and absorbance CER methods was determined by measuring several samples with both methods.

LCAT Plasmid Constructs. Human LCAT cDNA was optimized for expression and cloned into pcDNA4 containing a C-terminal 6-His tag (Glukhova et al., 2015). Mutants were made using the QuikChange II Site-Directed Mutagenesis kit (Stratagene, San Diego, CA). Primer sequences are provided in Supplemental Table 1.

Expression of rhLCAT in Tissue Culture Cells. Wild-type (WT) or mutant LCAT plasmid (with a C-terminal 6-His tag) was transfected into freshly thawed human embryonic kidney 293 (HEK293) cells using TrueFect-Lipo Transfection Reagent (United Biosystems, Herndon, VA) in Opti-MEM media (Life Technologies, Carlsbad, CA). Twenty-four hours later, cells were checked for efficiency of transfection by green fluorescent protein expression. The medium was then changed to serum-free Dulbecco's modified Eagle's medium containing antibiotics, glutamine, and $3.75 \mathrm{mM}$ valproic acid sodium salt (Sigma, St. Louis, MO). The conditioned medium was removed 48-72 hours later, centrifuged for 5 minutes at $500 \mathrm{~g}$ at $4^{\circ} \mathrm{C}$, and stored frozen at $-80^{\circ} \mathrm{C}$ until use.

Continuous Fluorescence Assay for LCAT. A novel, sensitive fluorescent 96-well plate assay for LCAT, using methylumbelliferyl 
palmitate (MUP) as a substrate, was developed to measure the phospholipase activity of LCAT. The activity was measured by following the increase in fluorescence due to methylumbelliferone formation. Methylumbelliferone (M-1381; Sigma-Aldrich), prepared at $100 \mathrm{mM}$ in dimethylsulfoxide (DMSO) and diluted further in phosphate-buffered saline, was used to generate standard curves. For apparent $K_{\mathrm{m}}$ and $\mathrm{V}_{\max }$ calculations, $1 \mathrm{ml}$ rhLCAT (Rousset et al., $2010)$ at $50 \mu \mathrm{g} / \mathrm{ml}$ in assay buffer (0.1 M sodium phosphate, $\mathrm{pH} 7.4)$ was incubated with $2.8 \mu \mathrm{l}$ compound A (10 mg/ml in DMSO) or just DMSO (vehicle) at $37^{\circ} \mathrm{C}$ for 1 hour. Twenty microliters of each reaction (LCAT minus compound A, LCAT plus compound A, or assay buffer) was then added to black 96-well plates (Greiner, Kremsmünster, Austria) in triplicate. During the incubation of LCAT with compound A, MUP stock $(1 \mathrm{mg} / \mathrm{ml})$ was prepared by adding $30 \mu \mathrm{l}$ Triton X-100 (American Bioanalytical, Natick, MA) to $1 \mathrm{mg}$ MUP in a glass vial and then adding DMSO to $1.0 \mathrm{ml}$. Immediately before use, the MUP stock solution was diluted in assay buffer to $0,0.01,0.02,0.04,0.06,0.1$, and $0.15 \mathrm{mM}$ MUP and then $180 \mu \mathrm{l}$ of each dilution was immediately added to the wells to start the reaction of LCAT with MUP. LCAT activity (methylumbelliferone formation) was measured by fluorescence at an excitation wavelength of $340 \mathrm{~nm}$ and an emission wavelength of $460 \mathrm{~nm}$. Background due to MUP self-hydrolysis ("assay buffer only" wells) was subtracted from each result. Specific activity (moles of MUP hydrolyzed per gram of LCAT) in conditioned media was determined by dividing moles of MUP hydrolyzed by LCAT mass, which was determined by an enzyme-linked immunosorbent assay (BioVendor, Asheville, NC). Apparent $K_{\mathrm{m}}$ and $V_{\max }$ values were calculated using GraphPad Prism software (GraphPad Software Inc., La Jolla, CA).

Reaction of Compound A with Methionine Sulfoxide Reductase $A$ and Glutathione and Analysis by Mass Spectrometry. Recombinant mouse methionine sulfoxide reductase A ( $\mathrm{msrA}$ ) was produced and purified as described (Kim et al., 2010), with the three cysteine residues not at the active site mutated to serine (C107S/C218S/C227S) to avoid complications from oxidation or disulfide formation (Lim et al., 2011). Ten microliters of the modified $\mathrm{msrA}$, at $0.5 \mathrm{mg} / \mathrm{ml}$ in $12.5 \mathrm{mM}$ Tris, $\mathrm{pH} 7.4$, was adjusted to $0.1 \mathrm{mg} / \mathrm{ml}$ in compound A by the addition of $0.1 \mu \mathrm{l}$ of $10 \mathrm{mg} / \mathrm{ml}$ compound A stock solution prepared in dimethylformamide. After 30-minute incubation at $37^{\circ} \mathrm{C}, 2 \mu \mathrm{l}$ of the reaction was injected onto a reversed-phase column with an autosampler set to $4^{\circ} \mathrm{C}$ (Agilent 1100 Series HPLC System; Agilent Technologies, Santa Clara, CA). The system contained a Zorbax 300-Å StableBond C18 MicroBore column (865630-902, $1.0 \times 50 \mathrm{~mm}$, $3.5 \mu \mathrm{m})$. The initial solvent was $0.1 \%$ formic acid with gradient elution by acetonitrile $/ 0.1 \%$ formic acid, increasing at $2 \% / \mathrm{min}$ with a flow rate of $20 \mu \mathrm{l} / \mathrm{min}$. Positive ion electrospray ionization mass spectra were obtained with an Agilent 6520 mass spectrometer equipped with a time-of-flight detector. The capillary voltage was $3500 \mathrm{~V}$, the fragmentor voltage was $235 \mathrm{~V}$, the nitrogen gas temperature was $350^{\circ} \mathrm{C}$, and data were collected in the mass range of $500-2500 \mathrm{~m} / z$. Mass spectra were analyzed and deconvoluted using MassHunter software (version B.05; Agilent Technologies).

For the reaction of compound A with glutathione (GSH), a solution of $100 \mu \mathrm{M}$ GSH was prepared in $50 \mathrm{mM}$ Tris and $1 \mathrm{mM}$ diethylenetriamine-pentaacetic acid, $\mathrm{pH}$ 7.5. One microliter of $10 \mathrm{mg} / \mathrm{ml}$ compound $\mathrm{A}$ in dimethylformamide was added to $99 \mu \mathrm{l}$ GSH and incubated at $37^{\circ} \mathrm{C}$ for 30 minutes, after which $1 \mu \mathrm{l}$ was injected into the same high-performance liquid chromatography (LC)mass spectrometry (MS) system described above. For this analysis, the instrument was run as a tandem mass spectrometer to sequence the modified GSH. The capillary voltage was $3500 \mathrm{~V}$, the fragmentor voltage was $175 \mathrm{~V}$, the nitrogen gas temperature was $300^{\circ} \mathrm{C}$, and the collision cell energy was ramped linearly from 9 to $69 \mathrm{~V}$ for the $\mathrm{m} / z$ range from 300 to 1500 .

Nuclear magnetic resonance (NMR) analysis was performed to examine the reaction of compound 5 [3-((5-(methylthio)-1,3,4-thiadiazol2-yl)thio)pyrazine-2-carbonitrile] with GSH. We added $2 \mathrm{ml}$ deuterated dimethyl formamide (dDMF) to a solution of GSH (3.3 mg, $10.7 \mathrm{mmol}$;
Sigma-Aldrich) in $1 \mathrm{ml} \mathrm{D}_{2} \mathrm{O}$ in an NMR tube at room temperature, and we then added compound $5(2.86 \mathrm{mg}, 10.7 \mathrm{mmol})$ (Table 1$)$. The resulting $2: 1 \mathrm{dDMF} / \mathrm{D}_{2} \mathrm{O}$ solution was left at room temperature for 48 hours with occasional shaking. The reaction proceeded cleanly, with no unreacted compound 5 detected. The reaction resulted in attachment of GSH to the third position of the pyrazine ring of compound $A$ and departure of the 5-(methylthio)-1,3,4-thiadiazole-2-thiol (thiadiazole ring) as the leaving group.

Reaction of Compound A with LCAT and Analysis by MS. Compound A was dissolved in DMSO to a concentration of $10 \mathrm{mg} / \mathrm{ml}$ and was then diluted to $1.0 \mathrm{mM}$ in phosphate-buffered saline, $\mathrm{pH}$ 7.4. Next, $1.5 \mu \mathrm{l}$ of $1.0 \mathrm{mM}$ compound A or vehicle was added to $15.0 \mu \mathrm{lhLCAT}$ at $1 \mathrm{mg} / \mathrm{ml}$ and incubated at $37^{\circ} \mathrm{C}$ for 30 minutes. LCAT, incubated with either vehicle or compound A, was deglycosylated under denaturing conditions using Protein Deglycosylation Mix (New England Biolabs, Ipswich, MA). $1.5 \mu \mathrm{l} \mathrm{H}_{2} \mathrm{O}$ and $2.0 \mu \mathrm{l} 10 \times$ glycoprotein denaturing buffer were added to each tube containing $15 \mu \mathrm{l} \mathrm{rhLCAT}$, and the reaction was heated at $100^{\circ} \mathrm{C}$ for 15 minutes. The reaction was chilled on ice and centrifuged for 10 seconds. $5.0 \mu \mathrm{l}$ $10 \times$ Glyco 2 buffer, $5 \mu \mathrm{l} 10 \% \mathrm{NP}-40$, and $15 \mu \mathrm{l} \mathrm{H}_{2} \mathrm{O}$ were added to each tube. After gentle mixing, $5.0 \mu \mathrm{l}$ deglycosylation enzyme cocktail was added. The solution was mixed again and incubated at $37^{\circ} \mathrm{C}$ for 4 hours and then subjected to SDS-PAGE on a $4 \%-12 \%$ Bis-Tris NuPAGE gel (Thermo Fisher Scientific/Invitrogen, Carlsbad, CA) and stained with SimplyBlue (Invitrogen). Gel pieces were destained with $200 \mathrm{mM}$ sodium bicarbonate/40\% ACN $(1 \times 400,1 \times 200 \mu \mathrm{l})$ with frequent vortexing and were dried in a SpeedVac treated with tributylphosphine and iodoacetamide using the ProteoPrep Reduction and Alkylation Kit (Sigma), washed twice with $25 \mathrm{mM} \mathrm{NH}_{4} \mathrm{CO}_{3}$ and then $200 \mu \mathrm{l} \mathrm{NH}_{4} \mathrm{CO}_{3}$ in $50 \%$ acetonitrile (ACN) for 15 minutes, and then desiccated in a SpeedVac.

For trypsin digestion, $20 \mu \mathrm{g}$ trypsin (sequencing grade; Promega, Madison, WI) was dissolved in $1.6 \mathrm{ml} 25 \mathrm{mM} \mathrm{NH}_{4} \mathrm{CO}_{3}\left(\right.$ in $\left.\mathrm{H}_{2} \mathrm{O}\right)$ and enough of the trypsin solution was added to just cover the gel pieces. Gel pieces were rehydrated on ice for 30 minutes and excess solution was removed. Then $25 \mathrm{mM} \mathrm{NH}_{4} \mathrm{CO}_{3}$ was added to cover the gel pieces. After a brief spin, the samples were placed in an incubator at $37^{\circ} \mathrm{C}$ overnight. After briefly spinning down the gel pieces, the supernatant (containing peptides) was transferred to a clean tube. Peptides remaining in the gel slices were extracted by incubating the gel pieces, with shaking, in extraction buffer (50\% acetonitrile, $5 \%$ formic acid) at room temperature for 30 minutes and collecting the supernatant (twice). All three supernatants containing tryptic peptides were then combined, concentrated in a SpeedVac, desalted using Ziptips (Millipore, Billerica, MA), and adjusted to $0.1 \%$ formic acid. The solution was subjected to analysis by LC/MS on an Orbitrap Fusion mass spectrometer coupled with an Ultimate 3000-nLC system (Thermo Fisher Scientific). Peptides were separated on an EASY-Spray $\mathrm{C}_{18}$ column $(75-\mu \mathrm{m} \times 25-\mathrm{cm}$ inner

TABLE 1

Structure of compound A and other LCAT activators

\begin{tabular}{|c|c|c|c|}
\hline Compound & Chemical Name & $\mathrm{EC}_{50}$ & ECmax \\
\hline & & $m M$ & $\%$ \\
\hline A & $\begin{array}{l}\text { 3-((5-(ethylthio)-1,3,4-thiadiazol-2- } \\
\text { yl)thio)pyrazine-2-carbonitrile }\end{array}$ & 0.794 & 161.3 \\
\hline 5 & $\begin{array}{l}\text { 3-((5-(methylthio)-1,3,4-thiadiazol-2- } \\
\text { yl)thio)pyrazine-2-carbonitrile }\end{array}$ & 5.81 & 149.6 \\
\hline 10 & $\begin{array}{l}\text { 3-[5-(3-cyano-pyrazin-2-ylsulfanyl)- } \\
\text { [1,3,4]thiadiazol-2-ylsulfanyl]- } \\
\text { propionamide }\end{array}$ & $142^{a}$ & $135.6^{b}$ \\
\hline 19 & $\begin{array}{l} \pm-(3 S, 4 R)-4-(3,5 \text {-difluorophenyl)-3- } \\
\quad \text { methoxy-1-(methylthio)azetidin-2-one }\end{array}$ & 1.69 & 167.3 \\
\hline 20 & $\begin{array}{l} \pm \text {-(3S,4R)-4-(4-bromophenyl)-3-methoxy-1- } \\
\quad \text { (methylthio)azetidin-2-one }\end{array}$ & 1.02 & 165.4 \\
\hline
\end{tabular}

EC50 and ECmax values are shown for compound A and derivatives (compounds 5 and 10) and for the $\beta$-lactam-based compounds 19 and 20.

${ }^{a}$ Calculated at the highest dose.

${ }^{b}$ Did not reach maximum. 

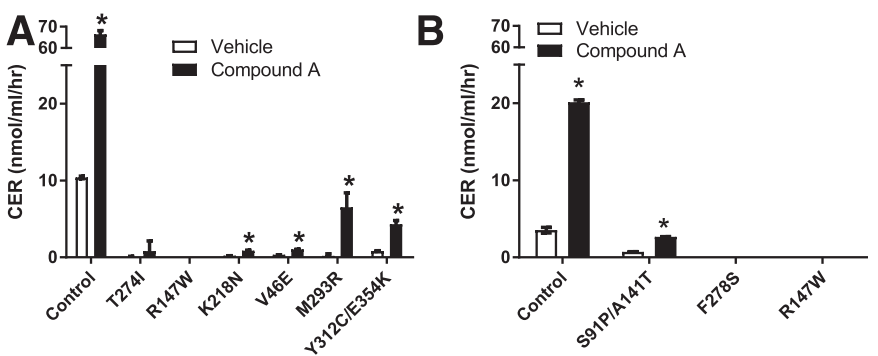

Fig. 1. Compound A increases LCAT plasma activity for some LCAT mutations. CER was determined in the plasma of patients with FLD and individuals with fish-eye disease using either (A) the ${ }^{3} \mathrm{H}$-CER method or (B) the CER enzymatic absorbance method. FLD mutations were T274I, R147W, K218N, and F278S. FED mutations were V46E, Y312C/E354K, and S91P/A141T. Results represent means \pm S.D. of at least triplicate measurements. ${ }^{*} P<0.05$ compared with the untreated control. FED, fisheye disease

diameter, $2-\mu \mathrm{m}$ particle size, and 100-Å pore size; Thermo Scientific). Separation was achieved by applying a 5\%-35\% linear gradient of acetonitrile plus $0.1 \%$ formic acid over 40 minutes at $300 \mathrm{nl} \cdot \mathrm{min}^{-1}$. An electrospray voltage of $1.9 \mathrm{kV}$ was applied to the eluent via the EASYSpray column electrode. The Orbitrap Fusion was operated in positive ion data-dependent mode. Full-scan $\mathrm{MS}^{1}$ was performed in the Orbitrap with a normal precursor mass range of $350-1500 \mathrm{~m} / z$ at a resolution of 120,000 . The automatic gain control target and maximum accumulation time settings were set to $4 \times 10^{5}$ and 150 milliseconds, respectively. $\mathrm{MS}^{2}$ was triggered by selecting the most intense precursor ions above an intensity threshold of $1 \times 10^{3}$ for collision-induced dissociation-MS ${ }^{2}$ fragmentation with an automatic gain control target and maximum accumulation time settings of $5 \times 10^{4}$ and 250 milliseconds, respectively. Mass filtering was performed by the quadrupole with a $1.2-\mathrm{m} / z$ transmission window, followed by collision-induced dissociation fragmentation in the Orbitrap at a resolution of 15,000 and a normalized collision energy of $37 \%$. To improve the spectral acquisition rate, parallelizable time was activated. The number of $\mathrm{MS}^{2}$ spectra acquired between full scans was restricted to a duty cycle of 3 seconds.

Raw data files were processed with Proteome Discoverer (version 1.4; Thermo Fisher Scientific), using the Sequest HT search node. Sequest HT searches were performed against the SwissProt human database (February 1, 2015). The mass tolerances for parent ions and fragment ions were set as $10 \mathrm{ppm}$ and $0.06 \mathrm{Da}$, respectively. Trypsin was set as the enzyme and a maximum of two missed cleavages was allowed. Carbamidomethylation of cysteine, methionine oxidation, deamidation (N,Q), and compound A $(103.0269 \mathrm{~m} / z)$ adduct on cysteine were selected as variable modifications. Peptide spectral matches were performed against a target decoy database. Peptide spectral matches were filtered with a false discovery rate cutoff of $1 \%$.

Synthesis of Compound A and Derivatives. Methods for synthesis of compound A and its derivatives, along with NMR spectra of synthesized compounds, are described in the Supplemental Material.

Molecular Modeling. Cholesterol, phospholipid (1-palmitoyl-2oleoyl-sn-glycero-3-phosphocholine), and compound A binding to LCAT were modeled using Maestro software (release 2015-3; Schrödinger, New York, NY). LCAT coordinates from a recent X-ray structure analysis (Protein Data Bank: 4X96) (Glukhova et al., 2015) were used for molecular docking, using the Glide application. Ligands were docked into the active site of LCAT, using flexible ligand sampling.

To evaluate the influence of Cys31 mutations or compound A and DTNB adducts on LCAT structure, a high-resolution LCAT structure was used (Protein Data Bank: 4XWG) (Piper et al., 2015). To prepare this structure, the Prime application was used to replace missing residues 231-237, the Tyr31 mutation was modified back to cysteine, and energy minimization was performed to relax the modified structure. This starting structure was then mutated in silico at

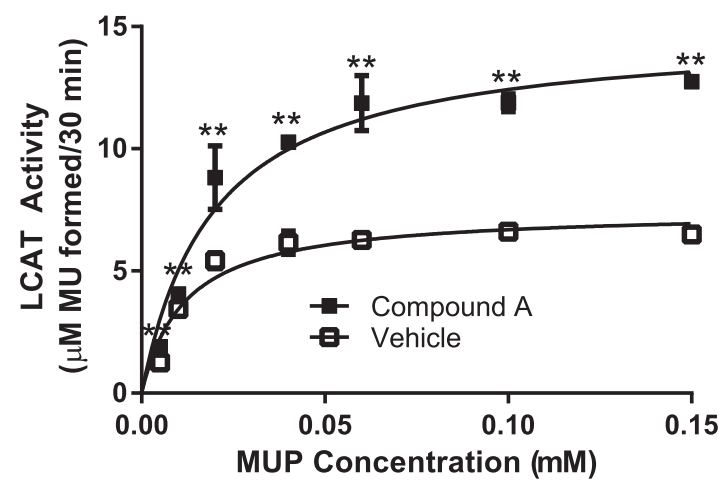

Fig. 2. Compound A increases the apparent $V_{\max }$ of LCAT. rhLCAT was incubated with compound A or vehicle. LCAT activity (100 ng LCAT/well in all reactions) was then measured by following methylumbelliferone (MU) formation at $460 \mathrm{~nm}$ after 30 minutes in the presence of increasing concentrations of the substrate, MUP. Results represent means \pm S.D. of at least triplicate measurements. ${ }^{* *} P<0.02$ compared with WT-LCAT treated under the same conditions.

Cys31 to simulate the various amino acid substitutions or adduct formation by compound A and DTNB. All-atom molecular dynamics simulations were then performed, using the Desmond application in SPC solvent with $0.15 \mathrm{M} \mathrm{NaCl}$ and at a temperature of $310 \mathrm{~K}$. Simulations were run for $20 \mathrm{~ns}$ on the National Institutes of Health High-Performance Computing Biowulf Cluster (Bethesda, MD).

All computations for the electrostatic potential of compound A were run using Gaussian 09 software (Gaussian, Inc., Wallington, CT). Visualizations were made using Gaussview (Gaussian, Inc.). A 6-31G* basis set was used to optimize the structure and calculate the electrostatic potential of compound A using the Hartree-Fock method.

Statistical Analysis. Unless otherwise indicated, all results are expressed as the mean \pm S.D. of at least triplicate measurements. Values were compared by a two-tailed $t$ test and $P$ values $<0.05$ were considered statistically significant.

\section{Results}

Naturally Occurring LCAT Mutations Activated by Compound A. Compound A and radiolabeled cholesterol were added to plasma from patients bearing different LCAT mutations to measure the CER. Figure 1 shows that compound A increased LCAT activity in healthy control plasma (WT) by 5 - to 6 -fold. The M293R LCAT homozygote and the compound LCAT heterozygotes Y312C/E354K and S91P/A141T had the highest response to compound A and reached activity levels that were at least 50\% of WT untreated plasma control samples. Two other LCAT mutations (namely, K218N and V46E) also showed considerable activation with compound A, but absolute activity levels of LCAT were still relatively low after compound A treatment. Mutations near the active site, which had no detectable baseline LCAT activity (R147W and F278S), showed no response to compound A.

Compound A Increases Apparent $K_{m}$ and $V_{\max }$ of LCAT by Interacting with Cysteine 31. Using MUP as a substrate to monitor the phospholipase step of the LCAT reaction, we tested the effect of compound $\mathrm{A}$ on the apparent $K_{\mathrm{m}}$ and $V_{\max }$ of recombinant LCAT (Fig. 2). Compound A had only a limited effect on the apparent $K_{\mathrm{m}}$ of LCAT but increased the apparent $V_{\max }$ by almost 2-fold (control: $7.47 \pm 0.26 \mu \mathrm{M}$ MU/30 minutes; compound A treatment: $14.9 \pm 0.6 \mu \mathrm{M}$ MU/30 minutes).

It was previously reported that Cys31 plays a role in modulating LCAT activity (Francone and Fielding, 1991; 


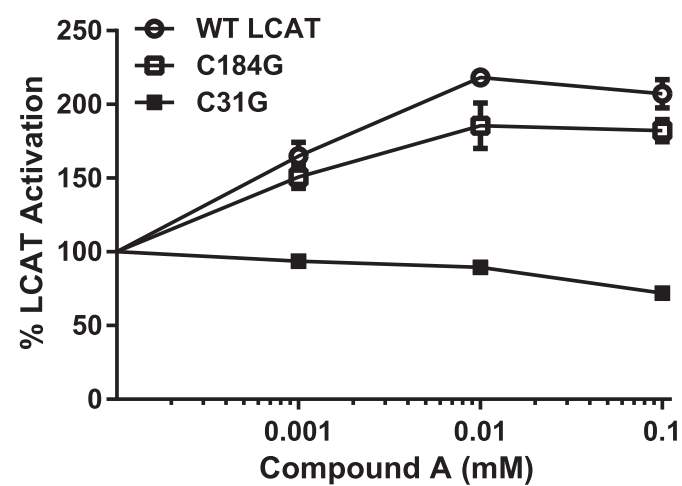

Fig. 3. Cys 31 is required for activation of LCAT by compound A. Conditioned media from cells transfected with WT-LCAT (Glukhova et al., 2015) and C31G and C184G mutants were incubated with the indicated concentrations of compound A ( $x$-axis) and then tested for LCAT activity with MUP as the substrate. Results are normalized to untreated activity levels for each LCAT variant. Results represent means \pm S.D. of at least triplicate measurements. ${ }^{* * *} P<0.005$ compared with WT-LCAT treated under the same conditions.

Qu et al., 1993; Kayser et al., 2013; Piper et al., 2015; Gunawardane et al., 2016). Dithiobis-nitrobenzoic acid (DTNB), a well-known inhibitor of LCAT (Supplemental Fig. 1), forms a mixed disulfide bond with Cys31, resulting in the addition of a nitrobenzoic acid group to Cys31 (Francone and Fielding, 1991). To test whether compound A, which has three sulfur groups (Supplemental Table S2), may also modulate LCAT activity by covalently reacting with Cys31, we used a codon-optimized fulllength human LCAT cDNA tagged with 6-His (Glukhova et al., 2015) and changed Cys31 to glycine (C31G) by site-directed mutagenesis. We also mutated Cys184 to glycine (C184G), because it is the only other free cysteine in LCAT. After transfection into cells, conditioned media from the cells were tested for LCAT activity, using MUP as the substrate in the presence and absence of compound A. As can be seen in Fig. 3, LCAT-C31G was not activated by compound A, whereas LCATC184G was activated to a similar extent as WT-LCAT.

Figure 4 shows that the replacement of Cys31 with either positively (Arg and Lys) or negatively (Glu) charged amino acids inhibits LCAT activity, consistent with the proposed mechanism for DTNB inhibition. In contrast, replacement of Cys31 with bulky hydrophobic amino acids (Trp, Tyr, Phe, Leu, and Met) resulted in a 2- to 3-fold activation of LCAT. By comparison, replacement of Cys31 with small polar amino acids (Ser and Thr) or His had a more limited effect (Fig. 4). These combined data are consistent with a model wherein compound A could activate LCAT by forming a hydrophobic adduct with Cys31.

Compound A Alkylates Thiols by Adding a Cyanopyrazine Moiety. To explore the reaction of compound A with proteins containing a free cysteine, we first investigated its effect on a mutated form of mouse msrA, which is $26 \mathrm{kDa}$ in size and contains a single, highly reactive low-pKa cysteine in its active site (Lim et al., 2012). Compound A was incubated with the mutated msrA and the reaction mixture was analyzed for a mass shift directly without any trypsinization on a quadrupole time-of-flight mass spectrometer. The major modified form of msrA found after 30-minute exposure to compound A gained +103.0 Da in mass and was stable to treatment with $25 \mathrm{mM}$ dithiothreitol (DTT) for 90 minutes. Based on the structure of compound A, this result

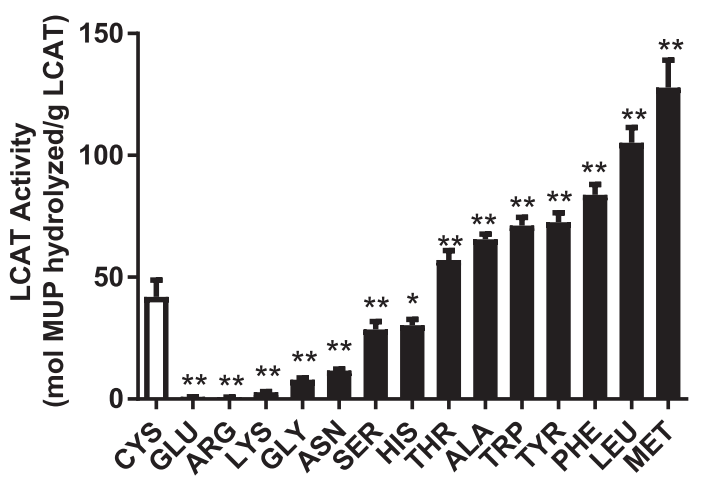

Amino Acid Replacement for Cys31

Fig. 4. Charged residues at position 31 inhibit and hydrophobic residues activate LCAT. Comparison of LCAT-specific activity of WT (Cys31) versus site-directed mutants at position 31 (x-axis). Conditioned media from HEK293 cells transfected with WT or mutant LCAT plasmids were collected and assayed for LCAT mass by enzyme-linked immunosorbent assay and LCAT activity using MUP as the substrate. Results represent means \pm S.D. of at least triplicate measurements. $* P<0.02$; $* * P<0.005$ compared with WT-LCAT. HEK293, human embryonic kidney 293

is consistent with an alkylation of the free cysteine of $\mathrm{msrA}$ with the cyanopyrazine ring from compound A (Fig. 5A), which is calculated to increase the mass by $103.02 \mathrm{Da}$.

Figure $5 \mathrm{~B}$ shows a similar experiment after incubating compound A with the tripeptide glutathione, which also contains a single highly reactive cysteine. A molar excess of glutathione readily reacted with compound $A$ to form a derivative with a monoisotopic mass of 410.1021 and another product of mass 177.9710 , which is compound A less the cyanopyrazine moiety. Tandem MS of the derivatized glutathione showed a difference of 103.0269 mass units, matching the mass difference in the $\mathrm{y} 2$ ion plus/minus the cyanopyrazine compound A adduct (Fig. 5B).

The reaction of glutathione with compound 5 (Supplemental Table S2), a derivative of compound A that also contains a cyanopyrazine ring and activates LCAT, was monitored by proton NMR (Fig. 6A). The resonance for one of two hydrogens $\left(\mathrm{H}_{\mathrm{a}}\right)$ on the cyanopyrazine ring, shifted downfield from 8.79 to $8.59 \mathrm{ppm}$, indicative of a more electron-withdrawing group (S-5membered thiadiazole ring) in the para position being replaced with a less electron-withdrawing group (glutathione $S$ ). The entire NMR spectrum for this reaction is shown in Supplemental Fig. 2 and is consistent with the proposed alkylation reaction of glutathione with the cyanopyrazine ring of compound $\mathrm{A}$ and its derivatives, as shown in the scheme in Fig. 6B.

To gain further insight into how compound A reacts with cysteines, we calculated the relative electrostatic potential of compound A by the Hartree-Fock method (Fig. 7, A and B). The carbon residue (atom $\mathrm{C}_{3}$, Fig. 7A) in the cyanopyrazine ring that alkylates Cys31 of LCAT was predicted to be the most electropositive part of the molecule $(+0.433)$ (see also Table 2). This carbon, which is connected to an electronwithdrawing $S$-thiadiazole group, is also at the end of an $\alpha, \beta$-unsaturated conjugated $\pi$-system due to the presence of the cyano group on the cyanopyrazine ring of compound A. This likely explains the electropositivity of this site and its susceptibility to nucleophilic attack by reactive cysteine residues in msrA and GSH. The charges on the analogous carbons in compounds 5 and 10 are similar $(+0.414)$ (Fig. 7, C and D; Table 2). 


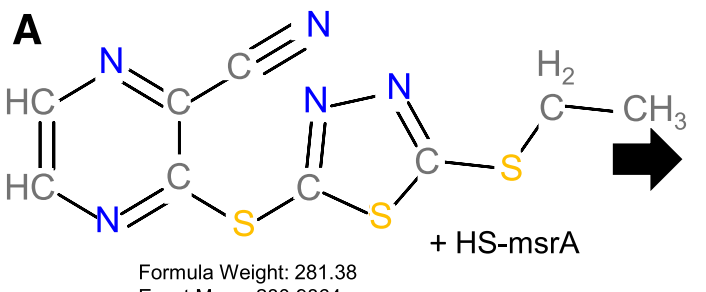<smiles>CCSc1nnc(Sc2nccnc2C#N)s1</smiles>
Exact Mass: 280.9864 Formula: $\mathrm{C}_{9} \mathrm{H}_{7} \mathrm{~N}_{5} \mathrm{~S}_{3}$

B MS-MS of the Glutathione-Compound A Product L product

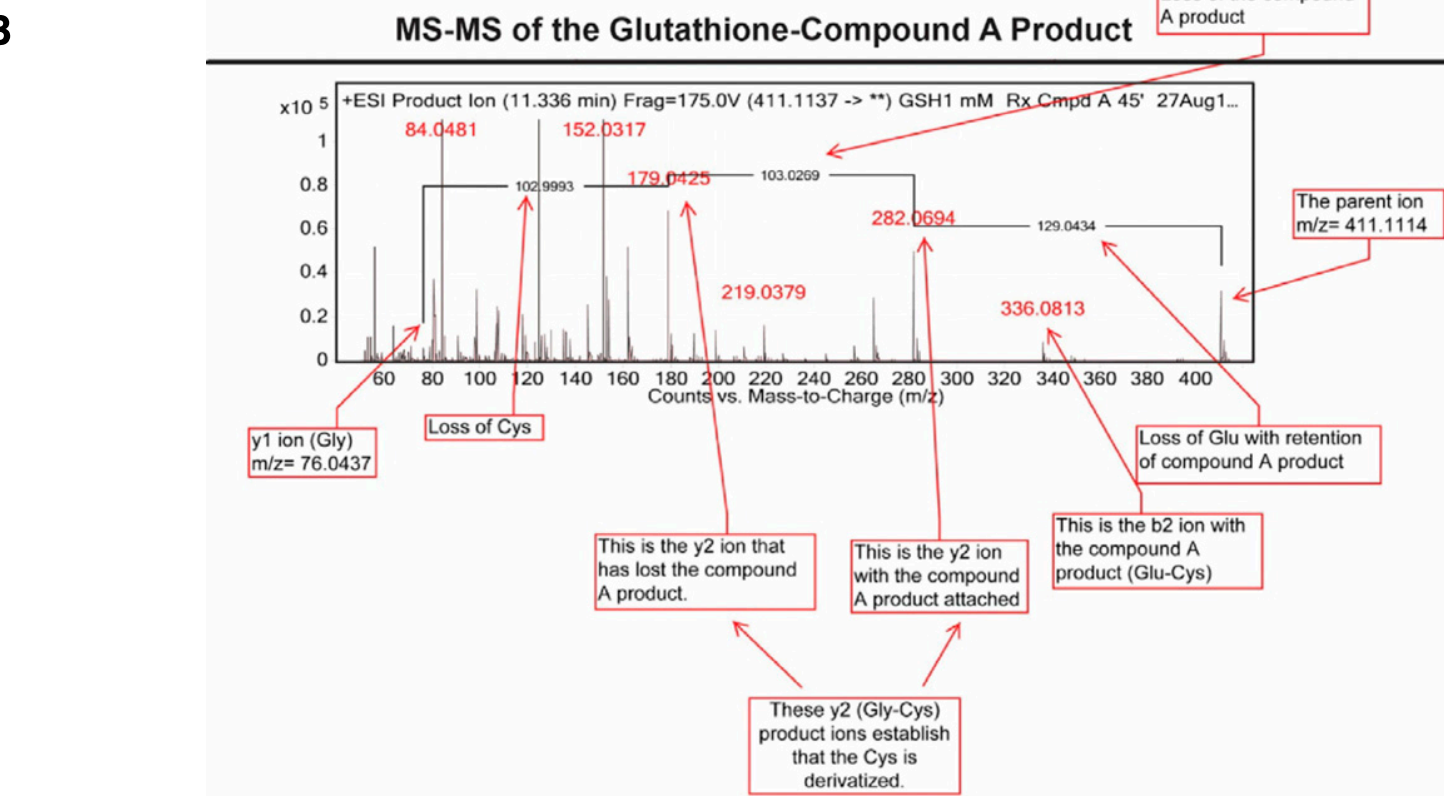

Fig. 5. MS analysis of msrA- and glutathione-compound A adducts. (A) Scheme for the reaction of the single cysteine residue on msrA with compound A. The major modified form of msrA gained +103.0 Da in mass, consistent with addition of the cyanopyrazine ring from compound A to the free cysteine of msrA. (B) Tandem mass spectrum of the glutathione cysteine reaction product demonstrates a cyanopyrazine ring. A molar excess of glutathione reacted readily with compound A to form a derivative with a monoisotopic mass of 410.1021 and another product of mass 177.9710 , which has the same mass as compound A less the cyanopyrazine moiety [see (A)]. Boxes indicate the position of various mass fragment ions from the reaction. ESI, electrospray ionization; HS-msrA; mutated msrA with a single sulfydryl group.

In Fig. 8, rhLCAT was incubated with compound A or vehicle and then deglycosylated, gel purified, and trypsinized. Tryptic peptides were analyzed by LC/MS on an Orbitrap Fusion mass spectrometer. In the $\mathrm{MS}^{2}$ mode, the monoisotopic $\mathrm{m} / \mathrm{z}$ of the $\mathrm{z}=4$ LCAT peptide fragments containing Cys31 increased from 640.8445 to 666.5986 after incubation with compound A (Fig. 8, A and B). This corresponds to a mass difference of $103.0164 \mathrm{Da}$, nearly identical to the size shift seen with msrA and GSH after incubation with compound A. The adduct was present only on LCAT incubated with compound A and was not detected in LCAT incubated with vehicle. In the $\mathrm{MS}^{3}$ mode, the $\mathrm{b}_{16}^{+2}$ fragment $\mathrm{m} / \mathrm{z}$ of the peptide containing Cys31 increased after incubation with compound A from 844.94153 to 896.45148 , or a mass difference of 103.01990 (Fig. 8, C and D). Consistent with the formation of a cyanopyrazine adduct with an S-C bond rather than a disulfide bond, DTT did not reverse LCAT activation by compound A (Supplemental Fig. 3).

Overall, the combined MS and NMR data indicate that the sulfhydryl group of Cys31 of LCAT acts as a nucleophile to attack compound $\mathrm{A}$, resulting in its alkylation by the attachment of a cyanopyrazine group to the side chain of Cys31.

Molecular Modeling of WT-LCAT Treated with Compound A and LCAT Mutants. Recent X-ray structural analyses of LCAT (Glukhova et al., 2015; Piper et al.,
2015; Gunawardane et al., 2016) reveal that Cys31 lies near the catalytic triad active site, with its backbone nitrogen contributing to the oxyanion hole. Therefore, to gain more insight on how WT-LCAT and its variants are altered by compound A, we performed molecular modeling studies.

First, we showed that both LCAT substrates, cholesterol and phosphatidylcholine, were able to dock in the active site of WT-LCAT in an orientation that would be expected to promote their catalysis (Supplemental Fig. 4, A and B). Compound A docked in the active site as well, with its cyanopyrazine ring positioned close to the sulfhydryl group of Cys31, which would facilitate their reaction (Fig. 9A; Supplemental Fig. 4C). A close-up view of the WT active site docked with phosphatidylcholine also shows how adding a hydrophobic adduct on Cys31 or replacing Cys31 with an amino acid with a hydrophobic side chain could potentially enhance phospholipid binding by increasing hydrophobic interactions with the glycerol backbone of phosphatidylcholine (Fig. 9B).

For LCAT-C31E (Supplemental Fig. 4D), which is catalytically inactive, the positively charged choline head group of phosphatidylcholine was predicted to be located near the negative side chain of glutamic acid in position 31 (Supplemental Fig. 4E), thus preventing the proper orientation of the phospholipid substrate for catalysis (Supplemental Fig. 4, compare D with B). 
A

Compound $5(t=0)$

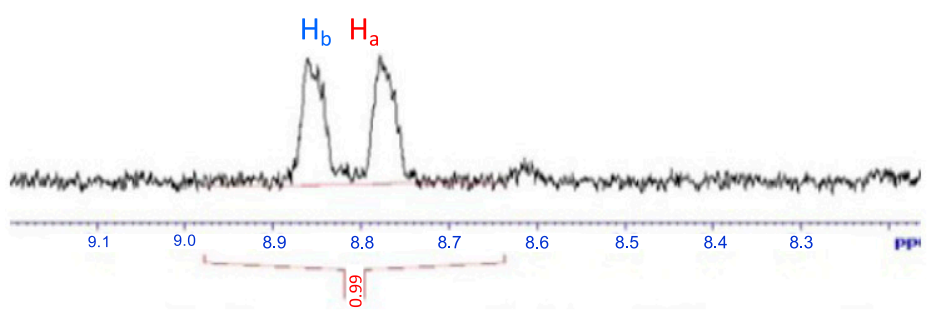

Compound $5+$ Glutathione (mid-reaction)

\section{Cmpd 5 Cmpd 5 - Glutathione Adduct}

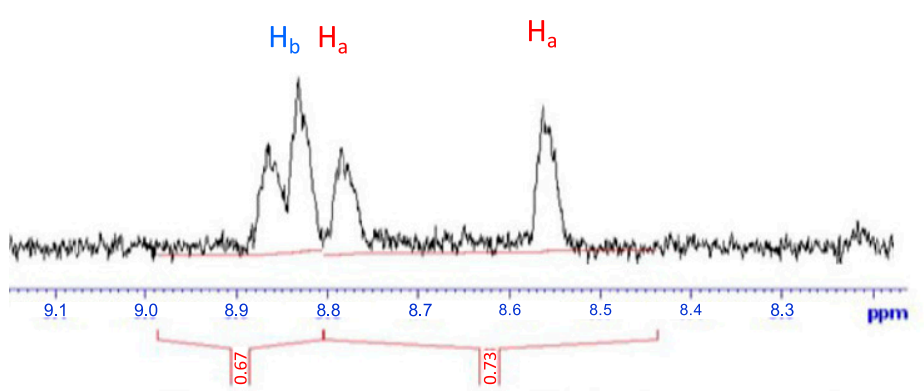

Compound $5+$ Glutathione (end of reaction)

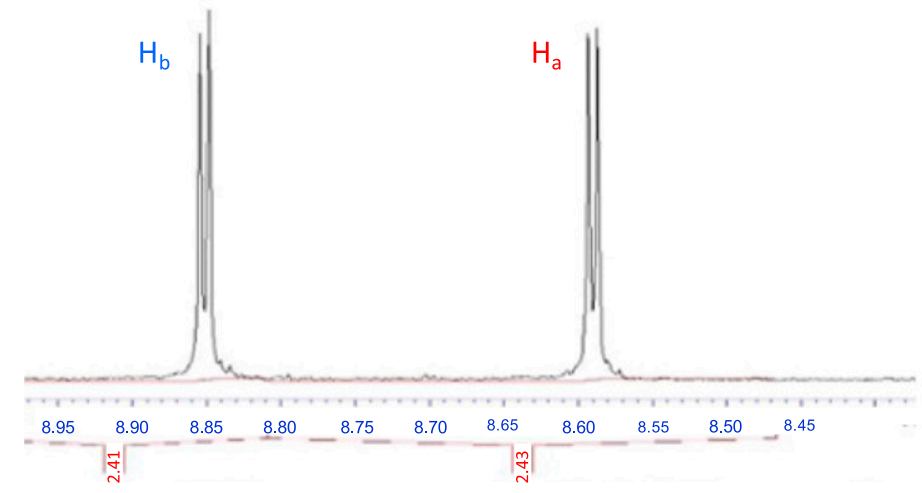

\section{Compound 5-Glutathione Adduct}<smiles>Cc1nc(C#N)c(SC[C@H](NC(=O)CCC(N)C(=O)O)C(=O)NCC(=O)O)nc1C</smiles>

B
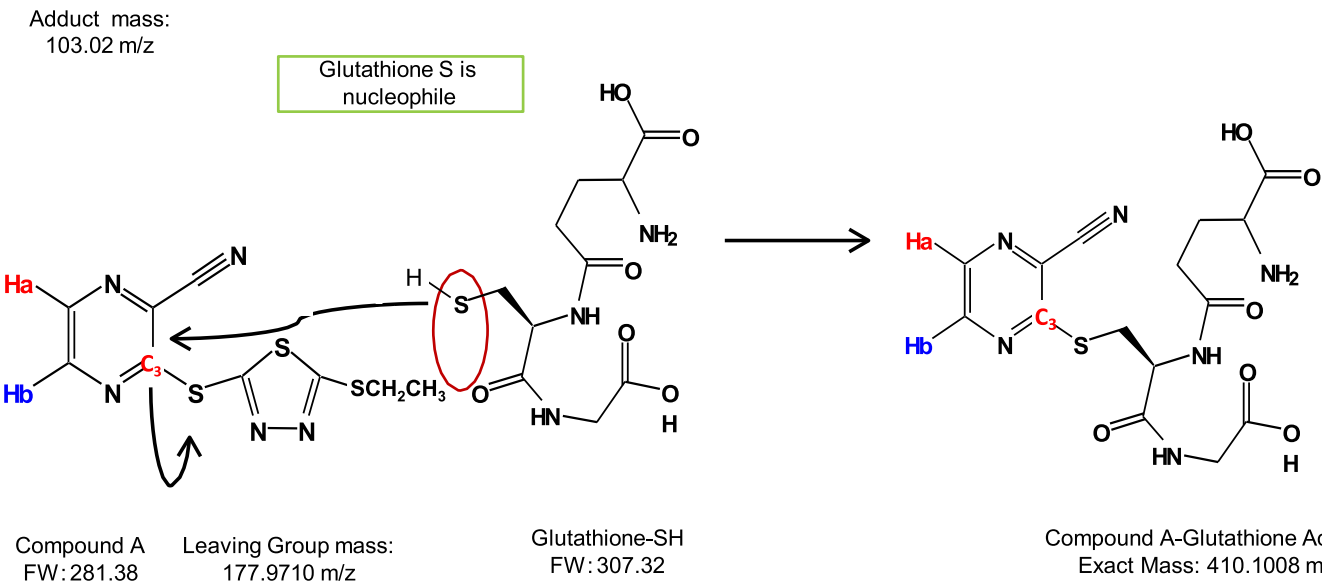

Compound A-Glutathione Adduct Exact Mass: $410.1008 \mathrm{~m} / \mathrm{z}$

Fig. 6. NMR analysis of the compound 5-glutathione adduct. (A) NMR spectra (left) of the region corresponding to two hydrogen groups $\left(\mathrm{H}_{\mathrm{a}}\right.$ and $\left.\mathrm{H}_{\mathrm{b}}\right)$ of compound 5 before the reaction (top), midreaction (24 hours; middle), and after completion of reaction (48 hours; bottom) with glutathione, showing a shift in the spectra for $\mathrm{H}_{\mathrm{a}}$ after derivitization with GSH. (B) Scheme for reaction mechanism of GSH with compound A. Carbon 3 of compound A (FW 281.38) undergoes nucleophilic attack by the free sulfhydryl group of glutathione-SH (FW 307.32), leading to a compound A-GSH adduct of 410.1008 $m / z$. FW, Formula weight. 
Next, we performed all-atom molecular dynamics simulations on WT-LCAT and several site-directed mutants, as well as WT-LCAT modified by compound A and DTNB. The various changes in LCAT structure were made in silico, using WT-LCAT as the starting structure, and were then allowed to relax in water at $37^{\circ} \mathrm{C}$ for $20 \mathrm{~ns}$ to reach a stable state (Supplemental Fig. 5). As can be seen in Fig. 10A, the simulation predicted that WT-LCAT undergoes considerable motion in the backbone of the membrane binding domain (residues 36-101), which is adjacent to the active site (Glukhova et al., 2015). Another dynamic region was predicted
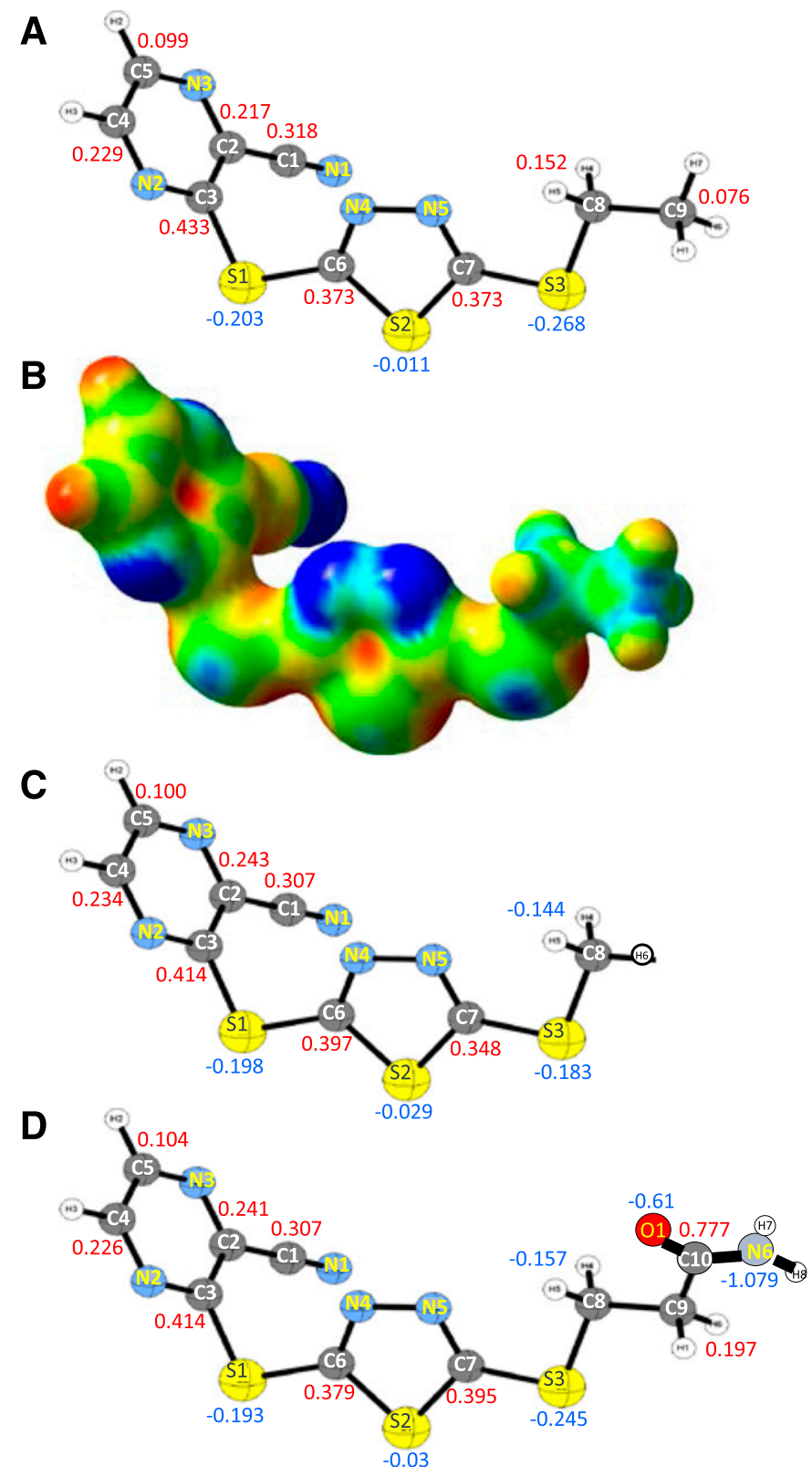

Fig. 7. Electrostatic potential map of compounds A, 5, and 10. (A) Charge distribution of compound A, numbered ball and stick view. Atomic charges on carbon (gray) and sulfur (yellow) are shown. Nitrogen atoms are shown in teal. Units for the atomic charge are relative units to the total charge of the molecule. (B) Electrostatic potential map of compound A. Blue shows electronegativity and red shows electropositivity. (C and D) Charge distribution of compound 5 (C) or compound 10 (D), numbered ball and stick view, as in (A). to be the lid region (residues 225-248) (Fig. 10A), which is near residues in the cap domain that help shape the substrate binding pocket (Glukhova et al., 2015; Piper et al., 2015). When the root-mean-square fluctuation difference in the backbone motion of LCAT modified with compound A or DTNB versus unmodified LCAT was calculated, several positions within the membrane binding domain and the lid and substrate binding pocket region showed differential responses to the modifications (Fig. 10B). A similar analysis was performed for various Cys31 mutants (C31M, C31E, C31F, C31L) that either activated or inhibited LCAT (Fig. 4) and a plot correlating the backbone motion with LCAT activity was made (Fig. 10C). This plot showed that areas of increased motion in the membrane binding domain, especially residues $83-90$, positively correlate with increased activity. In contrast, increased motion around the lid region, particularly amino acids 238-242, negatively correlated with LCAT activity. Overall, these results suggest that adduct formation by compound A on LCAT modifies its dynamic structure in particular regions of LCAT that lead to enhanced activity.

Effect of Compound A Derivatives on LCAT Activation. To determine the structural requirements of compound A for LCAT activation, we made and tested several derivatives of compound A (Table 1; Supplemental Table S2).

First, the alkyl substituent of the alkylthio group on the thiadiazole ring of compound A was changed from an ethyl to a methyl group, forming compound 5 (Supplemental Fig. 6A, scheme 1, Supplemental Table S2). Compound 5 retained LCAT activation but its $\mathrm{EC}_{50}$ was increased and its $\mathrm{EC}_{\max }$ was reduced compared with compound A (Table 1). Replacement of the thiadiazole ring of compound $\mathrm{A}$ with other groups, such as thiophene (Supplemental Fig. 6A, scheme 1, compound 6), as well as substituted benzenes (Supplemental Fig. 6A, scheme 1 , compounds 7 and 8), negated the ability of these derivatives to activate LCAT. In fact, compounds $6-8$ were inhibitory at high concentrations $\left(\mathrm{IC}_{50}>0.06\right)$. This is most likely due to

TABLE 2

Atomic charge from electrostatic potential of individual atoms of compounds A, 5 , and 10

\begin{tabular}{lccc}
\hline & \multicolumn{3}{c}{ Atomic Charge } \\
\cline { 2 - 4 } (Charge Distribution Numbering) & Compound A & Compound 5 & Compound 10 \\
\hline $\mathrm{N}_{1}$ & -0.45 & -0.448 & -0.449 \\
$\mathrm{C}_{1}$ & 0.318 & 0.307 & 0.307 \\
$\mathrm{C}_{2}$ & 0.217 & 0.243 & 0.241 \\
$\mathrm{C}_{3}$ & 0.433 & 0.414 & 0.414 \\
$\mathrm{~N}_{2}$ & -0.484 & -0.482 & -0.481 \\
$\mathrm{C}_{4}$ & 0.229 & 0.234 & 0.226 \\
$\mathrm{C}_{5}$ & 0.099 & 0.1 & 0.104 \\
$\mathrm{~N}_{3}$ & -0.382 & -0.391 & -0.39 \\
$\mathrm{~S}_{1}$ & -0.203 & -0.198 & -0.193 \\
$\mathrm{C}_{6}$ & 0.373 & 0.397 & 0.379 \\
$\mathrm{~N}_{4}$ & -0.364 & -0.396 & -0.379 \\
$\mathrm{~N}_{5}$ & -0.262 & -0.207 & -0.224 \\
$\mathrm{C}_{7}$ & 0.373 & 0.348 & 0.395 \\
$\mathrm{~S}_{2}$ & -0.011 & -0.029 & -0.03 \\
$\mathrm{~S}_{3}$ & -0.268 & -0.183 & -0.245 \\
$\mathrm{C}_{8}$ & 0.152 & -0.144 & -0.157 \\
$\mathrm{C}_{9}$ & 0.076 & & 0.197 \\
$\mathrm{C}_{10}$ & & & 0.777 \\
$\mathrm{~N}_{6}$ & & & 1.079 \\
$\mathrm{O}_{1}$ & & & -0.61 \\
\hline
\end{tabular}

Charge distribution for carbon, nitrogen and sulfur atoms of compounds A, 5 and 10 are shown. Units for the atomic charge are relative units to the total charge of the molecule. See Fig. 7 for compound numbering schemes. 


\section{A}
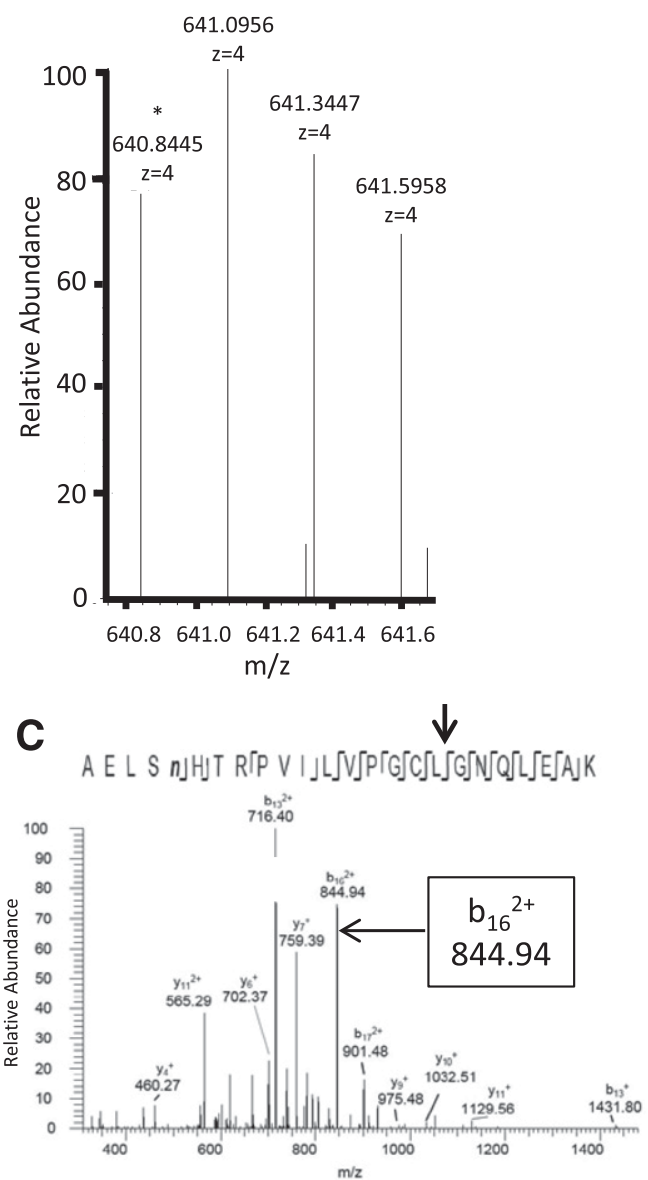

B LCAT + Compound A

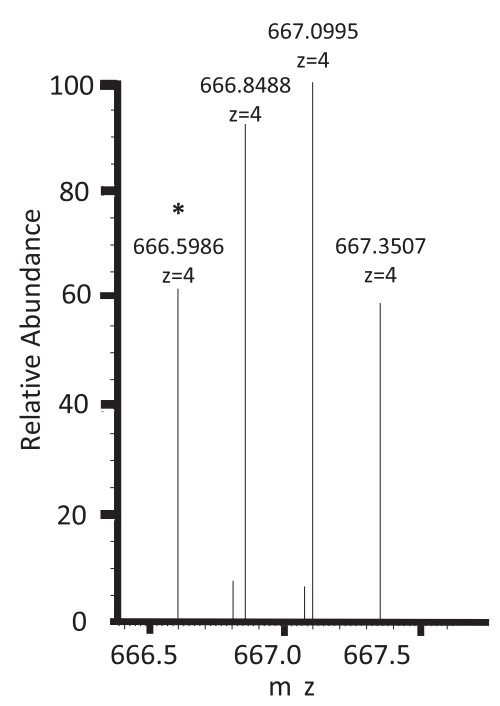

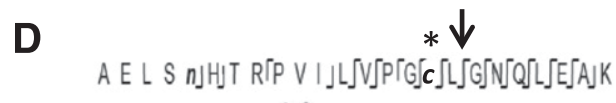

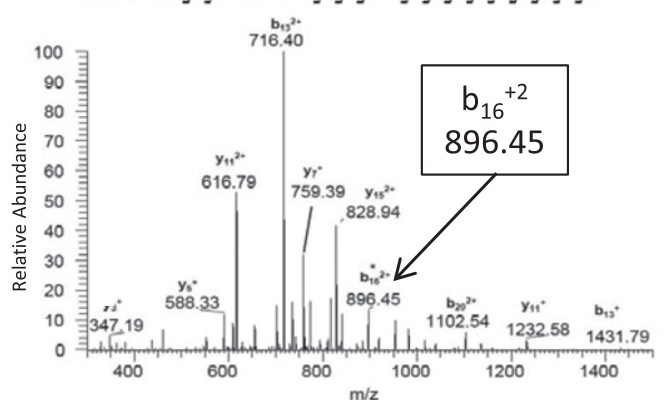

Fig. 8. MS analysis of LCAT treated with compound A. $\mathrm{MS}^{2}$ spectra of LCAT before (A) and after (B) treatment with compound A. Peaks correspond to precursor ions of the deamidated LCAT peptide AELSnHTRPVILVPGCLGNQLEAK, containing Cys31. The asterisk indicates the monoisotopic peak. $(\mathrm{C}, \mathrm{D}) \mathrm{MS}^{3}$ spectra produced by collision-induced dissociation of the $(\mathrm{M}+4 \mathrm{H})^{4+}$ precursor ions of the deamidated peptide AELSnHTRPVILVPGCLGNQLEAK, containing Cys31 before (C) and after (D) treatment with compound A. The arrow indicates the fragmentation position of LCAT peptide to produce the $b_{16}^{+2}$ fragment.

the lack of Lewis bases, such as nitrogen atoms, on the group attached to position 3 of the pyrazine ring. The presence of atoms that are Lewis bases might be necessary for an appropriate positioning of compound $\mathrm{A}$ in close proximity to the Cys31 (Supplemental Fig. 7), which is a more likely reason for lack of activity of compounds 6-8 (Supplemental Fig. 6A, scheme 1) rather than their ability to become good leaving groups.
Compounds substituting the electron-withdrawing $\mathrm{CN}$ group on the pyrazine ring of compound $\mathrm{A}$ with $\mathrm{CF}_{3}$ or $\mathrm{NO}_{2}$ groups and changing the pyrazine group to pyridine (Supplemental Fig. 6B, scheme 2, compounds 12-14), or situating the $\mathrm{CN}$ group on the opposite side of the ring compared with compound A (compound 15) also did not activate LCAT. In fact, compound 14 was also an inhibitor at high concentrations $\left(\mathrm{IC}_{50}=6.8 \mu \mathrm{M}\right.$ for compound 14). Furthermore, alteration of
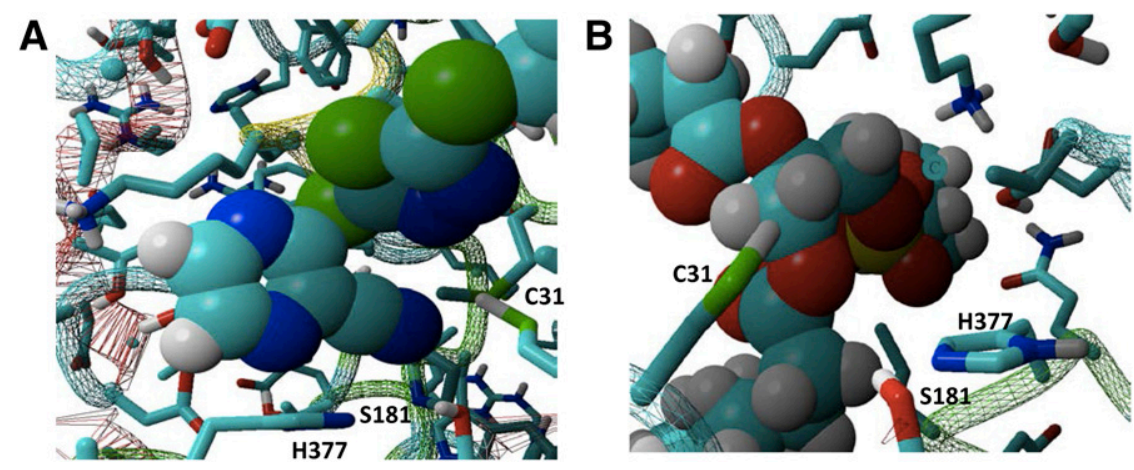

Fig. 9. Molecular modeling of the WT-LCAT active site. (A) WT-LCAT was docked with compound A in its active site, showing the close position of Cys31 near the cyanopyrazine ring of compound A. (B) WT-LCAT was docked with POPC in its active site, showing the close position of Cys31 near the hydrophobic glycerol backbone of POPC. His377 and Ser181 of the catalytic triad are also shown. POPC, 1-palmitoyl-2oleoyl-sn-glycero-3-phosphocholine. 
A

RMSF (Backbone)

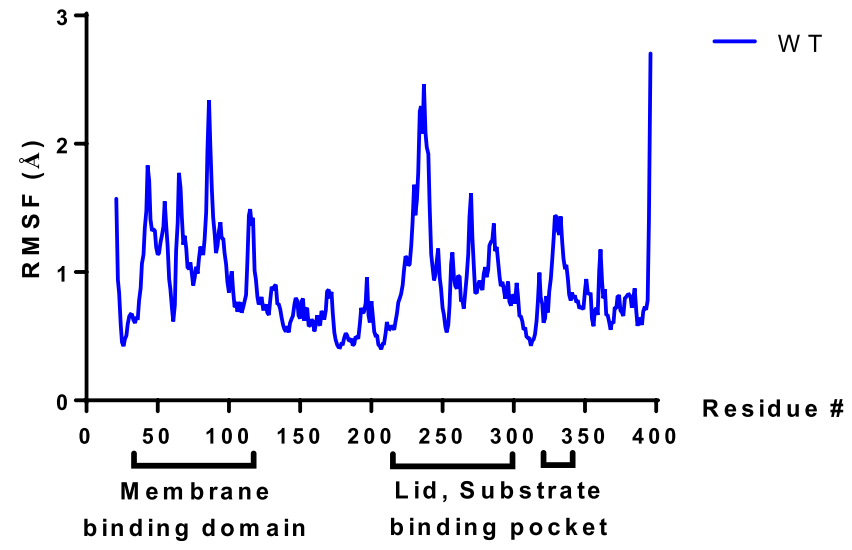

B

Delta RMSF (Backbone)

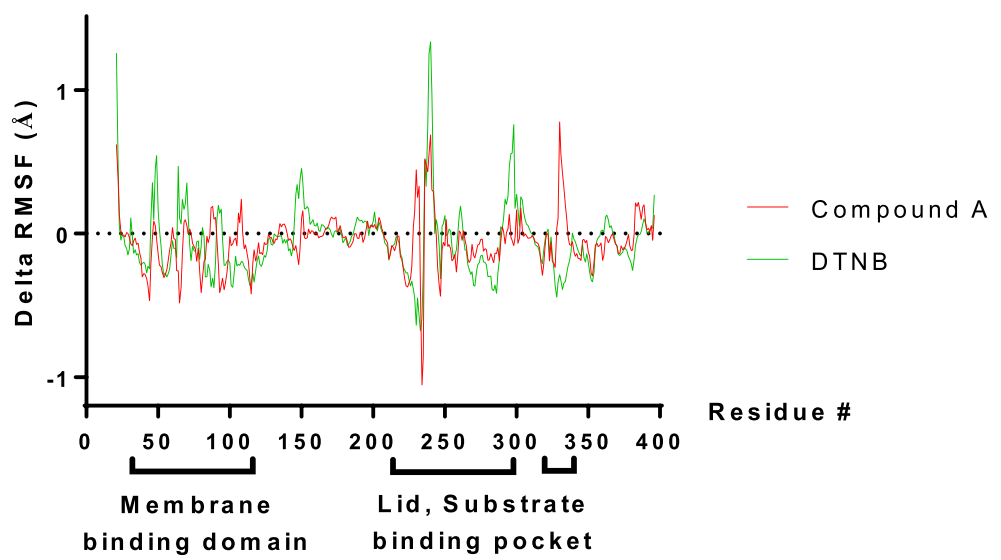

Fig. 10. Molecular dynamics simulations of LCAT reveal backbone motion. (A) RMSF (in Angstrom units) for WT-LCAT compared with the starting structure at each position in the LCAT sequence. (B) The $\triangle$ RMSF of WT-LCAT modified either with DTNB (green) or compound A (red) was calculated as the difference from the RMSF of WT-LCAT. (C) Correlation between $\triangle$ RMSF with LCAT activity, with a positive slope corresponding to protein regions in which increased backbone motion is associated with increased LCAT activity. Amino acid residues in the membrane binding domain (36-101) and the cap domain containing the lid (residues 226-246), as well as residues that help shape the substrate binding pocket (Glukhova et al., 2015; Piper et al., 2015), are shown below the $x$-axis. RMSF, root-mean-square fluctuation.

C

Slope Delta RMSF (Backbone)

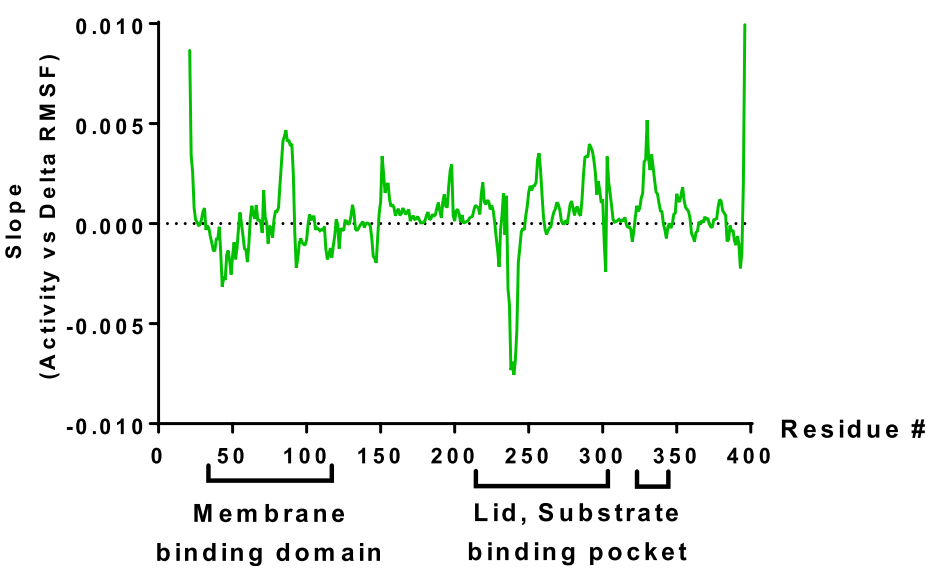

compound 13 (Supplemental Fig. 6B, scheme 2) by substituting the methylsulfanyl-[1,3,4]thiadiazol-2-ylsulfanyl group with a 4-nitro-phenylsulfanyl group (Supplemental Fig. 6B, scheme 2, compound 14) did not rescue its ability to activate LCAT. The lack of Lewis bases (e.g., electron-rich nitrogen atoms) as in compounds 17 and 18 (Supplemental Fig. 6C, scheme 3) led to compounds that were weak inhibitors of LCAT.
Next, we docked compound A with LCAT to investigate potential interactions with amino acid residues near the active site (Supplemental Fig. 7, A and B). The $S$-ethyl group attached to the thiadiazole ring was predicted to fit into and almost fully occupy a pocket within the substrate binding pocket that contains both hydrophobic and hydrophilic elements (Supplemental Fig. 7, A and B). We, therefore, redesigned compound A by replacing the $S$-ethyl tail with a 
propionamide group (Supplemental Fig. 6A, scheme 1, compound 10, Supplemental Table S2), so that it could potentially form hydrogen bonds with amino acid side chains in the active site (Supplemental Fig. 7, C and D). Compound 10, however, was only a relatively weak activator of LCAT compared with compound A (Table 1). Upon further inspection, the modeling revealed a potential clash between the propionamide group of compound 10 with Thr246 (Supplemental Fig. 7, C and D), possibly explaining its decreased potency (Table 1). Finally, a comparison of the docking of compound A (Supplemental Fig. 7, A and B) and compound 5 (Supplemental Fig. 7, E and F) with the LCAT active site revealed that compound $\mathrm{A}$, with its S- $\mathrm{CH}_{2}-\mathrm{CH}_{3}$ tail, fit into the pocket better than compound 5, with its shorter $\mathrm{S}_{-} \mathrm{CH}_{3}$ tail, which likely accounts for the superior LCAT activation by compound A (Table 1). For all three compounds, Cys31 and Thr248 were shown to interact with electron-rich nitrogen atoms (Lewis bases) in compound A and its derivatives.

Finally, we synthesized a novel class of thiol-reactive compounds based on monocyclic $\beta$-lactams (compounds 19 and 20, Supplemental Table S2; Supplemental Fig. 6D, scheme 4). Both of these compounds contain a methylthio group, which could form a disulfide bond with LCAT by $S$-methylating Cys31. Because the C31M mutation in LCAT had increased activity (Fig. 4), we predicted that these compounds would activate LCAT. Both of these compounds were nearly as good as compound A in activating LCAT (Table 1). In the case of compound 19, LCAT activation could be reversed with DTT, unlike compound A, consistent with compound 19 forming an adduct with LCAT by formation of a disulfide bond.

\section{Discussion}

A potential clinical indication for small-molecule LCAT activators (e.g., as compound A) is for the treatment of FLD. We previously investigated the use of rhLCAT as a potential therapy for FLD and found that it was safe and well tolerated (Shamburek et al., 2016a,b). Moreover, rhLCAT treatment almost completely normalized HDL-C levels, improved anemia, and stabilized renal function in a patient with FLD with advanced kidney disease (Shamburek et al., 2016a). rhLCAT, however, is an expensive biologic that requires repeated intravenous infusions. A small-molecule treatment approach would be a preferable therapy, if such a drug would activate the LCAT mutations that cause FLD. Compound A has already been shown to increase LCAT activity and raise HDL-C in the plasma of mice and hamsters (see Chen et al., 2012). We observed, in this study, that three of nine FLD mutations were activated by compound A to a level comparable to FLD heterozygotes. Because FLD heterozygotes are asymptomatic except for their low normal HDL-C levels, these results suggest that small-molecule LCAT activators could be a feasible therapeutic approach for a subset of patients with FLD, especially since the primary site of action of LCAT is in the plasma.

A second possible clinical indication for small-molecule LCAT activators is for the treatment of CVD. The strong negative correlation between plasma HDL-C levels and CVD risk and the ability of HDL to promote cholesterol efflux from cholesterol-loaded macrophages suggest that HDL-raising therapies might offer protection against CVD. To our knowledge, although some treatments to increase HDL-C, such as CETP inhibitors (Karathanasis et al., 2017), have not been successful in protecting against CVD, the possible efficacy of LCAT as a CVD therapeutic has not yet been tested. Studies of the role of LCAT, however, in the pathogenesis of atherosclerosis in both human and animal studies have yielded conflicting results (Rousset et al., 2011; Ahsan et al., 2014). A Mendelian randomization study of subjects with low HDL-C due to LCAT single nucleotide polymorphisms concluded that low levels of LCAT activity were not associated with an increased risk of CVD (Haase et al., 2012). In contrast, increased levels of pre- $\beta$-HDL and low LCAT activity were found in a cohort of patients with CVD (Sethi et al., 2010). In addition, some (Hovingh et al., 2005; Duivenvoorden et al., 2011; van den Bogaard et al., 2012) but not all (Calabresi et al., 2009) FLD heterozygotes appear to have increased carotid atherosclerosis (Ahsan et al., 2014). Moreover, intravenous rhLCAT infusion reduced atherosclerosis in rabbits (Zhou et al., 2009) and also improved plasma cholesterol efflux and raised plasma HDL-C levels in patients with CVD (Shamburek et al., 2016b). If ongoing clinical trials of rhLCAT demonstrate benefit in reducing atherosclerosis and/or in reducing clinical events in patients with CVD, this will likely greatly stimulate efforts to develop small-molecule activators of LCAT.

Another major finding from this study is that compound A forms a hydrophobic adduct with Cys31 of LCAT and increases the apparent $V_{\max }$ of LCAT for at least the first step of the reaction. Site-directed mutagenesis also showed that amino acids with hydrophobic side chains at residue 31 increased LCAT activity, whereas charged amino acid residues, both positive and negative, at the same position, strongly inhibited LCAT. These results are consistent with the qualitative findings for LCAT mutants first reported in the patent for compound A (Kayser et al., 2013). Replacing Cys31 with Tyr was also been previously described to enhance LCAT activity without significantly altering its structure (Piper et al., 2015). Interestingly, in chickens, the amino acid corresponding to Cys31 in human LCAT is the hydrophobic amino acid phenylalanine (Hengstschläger-Ottnad et al., 1995), and chickens have relatively high plasma levels of HDL-C. Consistent with our observation of decreased activity of the C31E mutation, DTNB, which adds a negatively charged adduct to Cys, is also known to inhibit LCAT (Francone and Fielding, 1991) (Supplemental Fig. 1). MS and NMR analysis of msrA and GSH incubated with compound A or its derivatives were all consistent with the presence of a hydrophobic cyanopyrazine adduct on the single cysteine in these two model compounds. Finally, MS of LCAT incubated with compound A revealed a $+103.016 \mathrm{~m} / z$ adduct on the tryptic peptide containing Cys31, thus confirming the addition of a cyanopyrazine ring to Cys 31 of LCAT.

Cys31 is located within the active site of LCAT (Glukhova et al., 2015); therefore, it is not unexpected that modification of this residue can affect LCAT activity. Like other interfacial enzymes, LCAT is unable to synthesize CE until it binds to a lipoprotein surface (Ahsan et al., 2014). The interaction with lipoproteins provides the substrates for LCAT but is also predicted to cause the displacement of the lid region that blocks the substrate binding pocket (Ahsan et al., 2014). By our modeling studies, adducts to Cys31 that activate LCAT or site-directed mutations in Cys31 that activate LCAT all show 
increased movement of the membrane binding domain, which may account for the increased LCAT activity after compound A treatment. Changes in the movement of other regions of LCAT, such as in the lid and substrate binding region, also correlated with changes in activity. From the crystal structures of WT-LCAT (Glukhova et al., 2015) and LCAT-C31Y (Piper et al., 2015), the cavity formed by the substrate binding pocket is relatively large and should be able to readily accommodate adducts at Cys31. As opposed to charge adducts at this position that inhibit LCAT (e.g. as occurs with DTNB), hydrophobic adducts appear to activate LCAT by promoting either the binding of its hydrophobic lipid substrates and/or a more favorable orientation of the lipid substrate for catalysis. Ongoing efforts to obtain the crystal structure of LCAT modified by compound A may provide further insight into the mechanism of LCAT activation.

None of the compound A derivatives that we synthesized showed improvements over the original compound first produced by Amgen in activating LCAT. In fact, many of them turned out to be inhibitors of LCAT at higher concentrations. This may have occurred because Cys31 is in close proximity to the catalytic triad (Ser181, Asp345, His377), particularly Ser181, which acts as a nucleophile in the first step of the LCAT reaction (Ahsan et al., 2014). This makes preparation of LCAT activators acting at Cys31 challenging, because they will have to be electrophiles that selectively react only with Cys31 and not with the active site serine residue. Therefore, alkylating reagents under future consideration should be relatively inactive toward oxygen nucleophiles like Ser. The fragment-based synthetic studies that we did on compound A revealed the importance of the presence of an $\alpha, \beta$-unsaturated system (cyanopyrazine ring), as well as the presence of Lewis bases (e.g., nitrogen atoms in the cyanopyrazine ring). The $S$-ethyl group tail of compound A also appears to be necessary for appropriately situating the molecule via hydrogen-bonding toward the Cys31 for nucleophilic attack.

A potential limitation of compound $\mathrm{A}$ is that its reaction with glutathione may indicate a lack of specificity. Many drugs that target sulfhydryl groups are in fact nonspecific and may react with reactive cysteines on other proteins (Zuniga et al., 2012). There are, however, many examples of sulfhydryltargeting drugs in current use. The reaction of compound $\mathrm{A}$ with LCAT may be somewhat specific, since the reaction with LCAT was complete at 30-60 minutes, whereas the reaction of compound A with GSH took 48 hours to complete. The Cys31 sulfhydryl in LCAT may be more reactive, possibly due to a lower $\mathrm{p} K_{\mathrm{a}}$ induced by the effect of neighboring residues in the three-dimensional structure. In general, the rate of reaction of a cysteine is known to be directly proportional to the fraction that is ionized to the thiolate and is controlled by the $\mathrm{p} K_{\mathrm{a}}$. The excellent fit of compound A inside the LCAT binding pocket may also add to its specificity for LCAT.

We did succeed in producing a new class of small-molecule activators of LCAT based on thiol-reactive $\beta$-lactams. Because of their reversibility after DTT treatment, these new compounds likely activate LCAT by forming a mixed disulfide with LCAT by $S$-methylating Cys31. Again, a limitation of this new class of compounds is that many drugs that target sulfhydryl groups are nonspecific and may react with reactive cysteines on other proteins (Zuniga et al., 2012). Previous studies of the $N$-methylthio class of lactams have shown that the $\mathrm{N}-\mathrm{S}$ bond is relatively resistant to cleavage by most types of nucleophiles but is susceptible to thiophiles (Woulfe et al., 1985; Shah and Cama, 1987). Moreover, $N$-thiolated lactams are also known to be nontoxic to mammalian cells (Revell et al., 2007). If the lactam ring of these compounds can be appropriately decorated to improve selectivity, perhaps by making it structurally resemble a lipid substrate, it may be possible to make specific LCAT activators based on this class of molecules.

In summary, we describe the mechanism for compound A activation of LCAT and show that some natural LCAT mutations can be considerably activated by compound $\mathrm{A}$, thus revealing a possible new treatment strategy for FLD. We also describe a new class of small-molecule activators of LCAT based on sulfhydryl-reactive $\beta$-lactams. These results provide important new insights into the design of future LCAT activators.

\section{Acknowledgments}

The authors thank Dr. Tim Hanusa for advice on the Hartree-Fock modeling studies and Dr. Alisa Glukhova for construction of the WT-LCAT-6-His pcDNA4 plasmid.

\section{Authorship Contributions}

Participated in research design: Freeman, Demosky, Konaklieva, Kuskovsky, Aponte, Ossoli, Gordon, Koby, Shen, Vaisman, Jadhav, Calabresi, Gucek, Levine, Remaley.

Conducted experiments: Freeman, Demosky, Konaklieva, Kuskovsky, Aponte, Ossoli, Koby, Levine.

Contributed new reagents or analytic tools: Konaklieva, Kuskovsky, Shamburek, Calabresi, Levine.

Performed data analysis: Freeman, Demosky, Konaklieva, Kuskovsky, Aponte, Ossoli, Gordon, Koby, Shen, Jadhav, Levine, Remaley.

Wrote or contributed to the writing of the manuscript: Freeman, Konaklieva, Aponte, Ossoli, Manthei, Tesmer, Levine, Remaley.

\section{References}

Adimoolam S and Jonas A (1997) Identification of a domain of lecithin-cholesterol acyltransferase that is involved in interfacial recognition. Biochem Biophys Res Commun 232:783-787.

Ahsan L, Ossoli AF, Freeman LA, Vaisman B, Amar MJ, Shamburek RD, and Remaley AT (2014) Role of lecithin: cholesterol acyltransferase in HDL metabolism and atherosclerosis, in The HDL Handbook: Biological Functions and Clinical Implications (Komoda T ed) 2nd ed, pp 159-194, Academic Press, London. Asztalos BF, Roheim PS, Milani RL, Lefevre M, McNamara JR, Horvath KV, and Schaefer EJ (2000) Distribution of ApoA-I-containing HDL subpopulations in patients with coronary heart disease. Arterioscler Thromb Vasc Biol 20:2670-2676.

Asztalos BF, Tani M, and Schaefer EJ (2011) Metabolic and functional relevance of HDL subspecies. Curr Opin Lipidol 22:176-185.

Calabresi L, Baldassarre D, Castelnuovo S, Conca P, Bocchi L, Candini C, Frigerio B, Amato M, Sirtori CR, Alessandrini P, et al. (2009) Functional lecithin: cholesterol acyltransferase is not required for efficient atheroprotection in humans. Circulation 120:628-635.

Chen Z, Wang SP, Krsmanovic ML, Castro-Perez J, Gagen K, Mendoza V, Rosa R, Shah V, He T, Stout SJ, et al. (2012) Small molecule activation of lecithin cholesterol acyltransferase modulates lipoprotein metabolism in mice and hamsters. Metabolism 61:470-481.

Czarnecka H and Yokoyama S (1996) Regulation of cellular cholesterol efflux by lecithin:cholesterol acyltransferase reaction through nonspecific lipid exchange. $J$ Biol Chem 271:2023-2028.

Dobiásová M (1983) Lecithin: cholesterol acyltransferase and the regulation of endogenous cholesterol transport. Adv Lipid Res 20:107-194.

Dobiásová M and Schützová M (1986) Cold labelled substrate and estimation of cholesterol esterification rate in lecithin cholesterol acyltransferase radioassay. Physiol Bohemoslov 35:319-327.

Duivenvoorden R, Holleboom AG, van den Bogaard B, Nederveen AJ, de Groot E, Hutten BA, Schimmel AW, Hovingh GK, Kastelein JJ, Kuivenhoven JA, et al. (2011) Carriers of lecithin cholesterol acyltransferase gene mutations have accelerated atherogenesis as assessed by carotid 3.0-T magnetic resonance imaging [corrected]. J Am Coll Cardiol 58:2481-2487.

Feister HA, Auerbach BJ, Cole LA, Krause BR, and Karathanasis SK (2002) Identification of an IL-6 response element in the human LCAT promoter. J Lipid Res 43:960-970.

Francone OL and Fielding CJ (1991) Effects of site-directed mutagenesis at residues cysteine-31 and cysteine-184 on lecithin-cholesterol acyltransferase activity. Proc Natl Acad Sci USA 88:1716-1720. 
Glukhova A, Hinkovska-Galcheva V, Kelly R, Abe A, Shayman JA, and Tesmer JJ (2015) Structure and function of lysosomal phospholipase A2 and lecithin:cholesterol acyltransferase. Nat Commun 6:6250.

Gordon SM and Remaley AT (2017) High density lipoproteins are modulators of protease activity: implications in inflammation, complement activation, and atherothrombosis. Atherosclerosis 259:104-113.

Gunawardane RN, Fordstrom P, Piper DE, Masterman S, Siu S, Liu D, Brown M, Lu M, Tang J, Zhang R, et al. (2016) Agonistic human antibodies binding to lecithincholesterol acyltransferase modulate high density lipoprotein metabolism. J Biol Chem 291:2799-2811.

Haase CL, Tybjærg-Hansen A, Qayyum AA, Schou J, Nordestgaard BG, and FrikkeSchmidt R (2012) LCAT, HDL cholesterol and ischemic cardiovascular disease: a Mendelian randomization study of HDL cholesterol in 54,500 individuals. J Clin Endocrinol Metab 97:E248-E256.

Hengstschläger-Ottnad E, Kuchler K, and Schneider WJ (1995) Chicken lecithincholesterol acyltransferase. Molecular characterization reveals unusual structure and expression pattern. J Biol Chem 270:26139-26145.

Holleboom AG, Kuivenhoven JA, Peelman F, Schimmel AW, Peter J, Defesche JC, Kastelein JJ, Hovingh GK, Stroes ES, and Motazacker MM (2011) High prevalence of mutations in LCAT in patients with low HDL cholesterol levels in The Netherlands: identification and characterization of eight novel mutations. Hum Mutat 32:1290-1298.

Hovingh GK, Hutten BA, Holleboom AG, Petersen W, Rol P, Stalenhoef A, Zwinderman AH, de Groot E, Kastelein JJ, and Kuivenhoven JA (2005) Compromised LCAT function is associated with increased atherosclerosis. Circulation 112:879-884.

Karathanasis SK, Freeman LA, Gordon SM, and Remaley AT (2017) The changing face of HDL and the best way to measure it. Clin Chem 63:196-210.

Kayser F, LaBelle M, Shan B, Zhang J, and Zhou M (2013), inventors, Amgen, Inc. assignee. Methods for treating atherosclerosis. U.S. patent 8426358. 2013 April 23

Kempen HJ, Gomaraschi M, Simonelli S, Calabresi L, Moerland M, Otvos J, Jeyarajah E, Kallend D, and Wijngaard PL (2016) Persistent changes in lipoprotein lipids after a single infusion of ascending doses of MDCO-216 (apoA-IMilano/ POPC) in healthy volunteers and stable coronary artery disease patients. Atherosclerosis 255:17-24.

Khovidhunkit W, Kim MS, Memon RA, Shigenaga JK, Moser AH, Feingold KR, and Grunfeld C (2004) Effects of infection and inflammation on lipid and lipoprotein metabolism: mechanisms and consequences to the host. $J$ Lipid Res 45: 1169-1196.

Kim G, Cole NB, Lim JC, Zhao H, and Levine RL (2010) Dual sites of protein initiation control the localization and myristoylation of methionine sulfoxide reductase A. J Biol Chem 285:18085-18094.

Krause BR and Remaley AT (2013) Reconstituted HDL for the acute treatment of acute coronary syndrome. Curr Opin Lipidol 24:480-486.

Lim JC, Gruschus JM, Kim G, Berlett BS, Tjandra N, and Levine RL (2012) A low $\mathrm{pKa}$ cysteine at the active site of mouse methionine sulfoxide reductase A. J Biol Chem 287:25596-25601.

Lim JC, You Z, Kim G, and Levine RL (2011) Methionine sulfoxide reductase A is a stereospecific methionine oxidase. Proc Natl Acad Sci USA 108:10472-10477.

Moraitis AG, Freeman LA, Shamburek RD, Wesley R, Wilson W, Grant CM, Price S, Demosky S, Thacker SG, Zarzour A, et al. (2015) Elevated interleukin-10: a new cause of dyslipidemia leading to severe HDL deficiency. J Clin Lipidol 9:81-90.

Ossoli A, Neufeld EB, Thacker SG, Vaisman B, Pryor M, Freeman LA, Brantner CA Baranova I, Francone NO, Demosky SJ, Jr, et al. (2016) Lipoprotein X causes renal disease in LCAT deficiency. PLoS One 11:e0150083.

Piper DE, Romanow WG, Gunawardane RN, Fordstrom P, Masterman S, Pan O, Thibault ST, Zhang R, Meininger D, Schwarz M, et al. (2015) The high-resolution crystal structure of human LCAT. J Lipid Res 56:1711-1719.

$\mathrm{Qu}$ SJ, Fan HZ, Blanco-Vaca F, and Pownall HJ (1993) Roles of cysteines in human lecithin:cholesterol acyltransferase. Biochemistry 32:3089-3094.

Rader DJ (2016) New therapeutic approaches to the treatment of dyslipidemia. Cell Metab 23:405-412.
Remaley AT, Amar M, and Sviridov D (2008) HDL-replacement therapy: mechanism of action, types of agents and potential clinical indications. Expert Rev Cardiovasc Ther 6:1203-1215.

Remaley AT, Norata GD, and Catapano AL (2014) Novel concepts in HDL pharmacology. Cardiovasc Res 103:423-428.

Revell KD, Heldreth B, Long TE, Jang S, and Turos E (2007) N-thiolated betalactams: studies on the mode of action and identification of a primary cellular target in Staphylococcus aureus. Bioorg Med Chem 15:2453-2467.

Rosenson RS, Brewer HB, Jr, Ansell BJ, Barter P, Chapman MJ, Heinecke JW, Kontush A, Tall AR, and Webb NR (2016) Dysfunctional HDL and atherosclerotic cardiovascular disease. Nat Rev Cardiol 13:48-60.

Rousset X, Shamburek R, Vaisman B, Amar M, and Remaley AT (2011) Lecithin cholesterol acyltransferase: an anti- or pro-atherogenic factor? Curr Atheroscler Rep 13:249-256.

Rousset X, Vaisman B, Auerbach B, Krause BR, Homan R, Stonik J, Csako G, Shamburek R, and Remaley AT (2010) Effect of recombinant human lecithin cholesterol acyltransferase infusion on lipoprotein metabolism in mice. J Pharmacol Exp Ther 335:140-148.

Rye KA and Barter PJ (2014) Cardioprotective functions of HDLs. J Lipid Res 55: $168-179$

Schwartz CC, VandenBroek JM, and Cooper PS (2004) Lipoprotein cholesteryl ester production, transfer, and output in vivo in humans. J Lipid Res 45:1594-1607.

Sethi AA, Sampson M, Warnick R, Muniz N, Vaisman B, Nordestgaard BG, TybjaergHansen A, and Remaley AT (2010) High pre-beta1 HDL concentrations and low lecithin: cholesterol acyltransferase activities are strong positive risk markers for ischemic heart disease and independent of HDL-cholesterol. Clin Chem 56: $1128-1137$.

Shah NV and Cama LD (1987) Synthesis of a novel carbapenem-potassium (5r,6r)1,1-difluoro-2-phenyl-6-(1r-hydroxyethyl)-carbapen-2-em-3-carboxylate. The use of a new N-protecting group in beta-lactam synthesis. Heterocycles 25:221-227.

Shamburek RD, Bakker-Arkema R, Auerbach BJ, Krause BR, Homan R, Amar MJ, Freeman LA, and Remaley AT (2016a) Familial lecithin:cholesterol acyltransferase deficiency: First-in-human treatment with enzyme replacement. J Clin Lipidol 10: 356-367.

Shamburek RD, Bakker-Arkema R, Shamburek AM, Freeman LA, Amar MJ, Auerbach B, Krause BR, Homan R, Adelman SJ, Collins HL, et al. (2016b) Safety and tolerability of ACP-501, a recombinant human lecithin:cholesterol acyltransferase, in a phase 1 single-dose escalation study. Circ Res 118:73-82.

Vaisman BL and Remaley AT (2013) Measurement of lecithin-cholesterol acyltransferase activity with the use of a peptide-proteoliposome substrate, in Lipoproteins and Cardiovascular Disease: Methods and Protocols, Methods in Molecular Biology (Freeman LA ed) pp 343-352, Springer, New York.

van den Bogaard B, Holleboom AG, Duivenvoorden R, Hutten BA, Kastelein JJ, Hovingh GK, Kuivenhoven JA, Stroes ES, and van den Born BJ (2012) Patients with low HDL-cholesterol caused by mutations in LCAT have increased arterial stiffness. Atherosclerosis 225:481-485.

Woulfe SR, Iwagami H, and Miller MJ (1985) Efficient N-sulfenylation of 2 -azetidinones using S-substituted thiophthalimides. Tetrahedron Lett $\mathbf{2 6}$ 3891-3894.

Zhou M, Sawyer J, Kelley K, Fordstrom P, Chan J, Tonn G, Carlson T, Retter M, Meininger, D, Cheng J, et al. (2009) Lecithin cholesterol acyltransferase promotes reverse cholesterol transport and attenuates atherosclerosis progression in New Zealand white rabbits (Abstract 5920). Circulation 120(Suppl 18):S1175.

Zuniga FI, Loi D, Ling KH, and Tang-Liu DD (2012) Idiosyncratic reactions and metabolism of sulfur-containing drugs. Expert Opin Drug Metab Toxicol 8:467-485

Address correspondence to: Alan T. Remaley, Lipid Metabolism Section, Cardiovascular and Pulmonary Branch, National Institutes of Health National Heart, Lung, and Blood Institute, Building 10, Room 2C433, 10 Center Drive, Bethesda, MD 20814. E-mail: aremaley1@mail.nih.gov 